Western University

Scholarship@Western

Brain and Mind Institute Researchers'

Publications

Brain and Mind Institute

$1-1-2014$

\title{
Bilateral saccadic deficits following large and reversible inactivation of unilateral frontal eye field.
}

Tyler R Peel

The Brain and Mind Institute, London, Ontario, Canada \& Graduate Program in Neuroscience, Western University, London, Ontario, Canada

Kevin Johnston

The Brain and Mind Institute, London, Ontario, Canada \& Department of Physiology and Pharmacology, Western University, London, Ontario, Canada \& Department of Psychology, Western University, London, Ontario, Canada

Stephen G Lomber

The Brain and Mind Institute, London, Ontario, Canada \& Department of Physiology and Pharmacology, Western University, London, Ontario, Canada \& Department of Psychology, Western University, London, Ontario, Canada \& Graduate Program in Neuroscience, Western University, London, Ontario, Canada

Brian D Corneil

The Brain and Mind Institute, London, Ontario, Canada \& Robarts Research Institute, London, Ontario, Canada \& Department of Physiology and Pharmacology, Western University, London, Ontario, Canada \& Department of Psychology, Western University, London, Ontario, Canada \& Graduate Program in Neuroscience, Western University, London, Ontario, Canada

Follow this and additional works at: https://ir.lib.uwo.ca/brainpub

Part of the Neurosciences Commons, and the Psychology Commons

\section{Citation of this paper:}

Peel, Tyler R; Johnston, Kevin; Lomber, Stephen G; and Corneil, Brian D, "Bilateral saccadic deficits following large and reversible inactivation of unilateral frontal eye field." (2014). Brain and Mind Institute Researchers' Publications. 127.

https://ir.lib.uwo.ca/brainpub/127 


\title{
Bilateral saccadic deficits following large and reversible inactivation of unilateral frontal eye field
}

\author{
Tyler R. Peel, ${ }^{1,5}$ Kevin Johnston, ${ }^{1,3,4}$ Stephen G. Lomber, ${ }^{1,3,4,5}$ and Brian D. Corneil ${ }^{1,2,3,4,5}$ \\ ${ }^{1}$ The Brain and Mind Institute, London, Ontario, Canada; ${ }^{2}$ Robarts Research Institute, London, Ontario, Canada; \\ ${ }^{3}$ Department of Physiology and Pharmacology, Western University, London, Ontario, Canada; ${ }^{4}$ Department of Psychology, \\ Western University, London, Ontario, Canada; and ${ }^{5}$ Graduate Program in Neuroscience, Western University, London, \\ Ontario, Canada
}

Submitted 31 May 2013; accepted in final form 17 October 2013

Peel TR, Johnston K, Lomber SG, Corneil BD. Bilateral saccadic deficits following large and reversible inactivation of unilateral frontal eye field. J Neurophysiol 111: 415-433, 2014. First published October 23, 2013; doi:10.1152/jn.00398.2013.-Inactivation permits direct assessment of the functional contribution of a given brain area to behavior. Previous inactivation studies of the frontal eye field (FEF) have either used large permanent ablations or reversible pharmacological techniques that only inactivate a small volume of tissue. Here we evaluated the impact of large, yet reversible, FEF inactivation on visually guided, delayed, and memory-guided saccades, using cryoloops implanted in the arcuate sulcus. While FEF inactivation produced the expected triad of contralateral saccadic deficits (increased reaction time, decreased accuracy and peak velocity) and performance errors (neglect or misdirected saccades), we also found consistent increases in reaction times of ipsiversive saccades in all three tasks. In addition, FEF inactivation did not increase the proportion of premature saccades to ipsilateral targets, as was predicted on the basis of pharmacological studies. Consistent with previous studies, greater deficits accompanied saccades toward extinguished visual cues. Our results attest to the functional contribution of the FEF to saccades in both directions. We speculate that the comparative effects of different inactivation techniques relate to the volume of inactivated tissue within the FEF. Larger inactivation volumes may reveal the functional contribution of more sparsely distributed neurons within the FEF, such as those related to ipsiversive saccades. Furthermore, while focal FEF inactivation may disinhibit the mirroring site in the other FEF, larger inactivation volumes may induce broad disinhibition in the other FEF that paradoxically prolongs oculomotor processing via increased competitive interactions.

frontal eye field; reversible inactivation; saccade generation

THE PRIMATE FRONTAL EYE FIELD (FEF) is a key brain area involved in the generation of saccadic eye movements (for review see Schall 2002). The functional role for the FEF in oculomotor control has been reinforced by a series of inactivation studies, which have described a triad of contralateral saccadic deficits [increased reaction time (RT), decreased accuracy and peak velocity] and performance errors (e.g., neglect, premature saccades, and an inability to maintain fixation) following permanent FEF ablations (Collin et al. 1982; Deng et al. 1986; Latto and Cowey 1971; Lynch 1992; Schiller 1980; Schiller and Chou 1998; van der Steen et al. 1986) or reversible pharmacological FEF inactivation (Dias et al. 1995; Dias and Segraves 1999; Shi et al. 1998; Sommer and Tehovnik 1997).

Address for reprint requests and other correspondence: B. D. Corneil, Robarts Research Inst., 100 Perth Dr., London, ON, Canada N6A 5K8 (e-mail: bcorneil@uwo.ca).
Each mode of inactivation has both advantages and disadvantages (Lomber 1999). Ablations lesion a large volume of tissue permanently, with assessments of oculomotor deficits occurring after weeks to months of recovery. The remaining oculomotor capabilities therefore reflect both what was lost due to the FEF lesion and the plastic capacity of the oculomotor network to recover over time. Reversible pharmacological inactivation is less invasive and enables study of the oculomotor system unconfounded by plastic recovery; however, the volume of inactivation is substantially smaller and varies with time as the drug diffuses and is metabolized. The effects of inactivating a large volume of the FEF on saccade behavior, unconfounded by recovery, remain unknown.

The goal of this study was to evaluate the FEF's contribution to visually guided, delayed, and memory-guided saccadic behavior via an assessment of oculomotor behavior before, during, and after large and reversible inactivation of unilateral FEF. To do this, we used the cryogenic inactivation technique (Lomber et al. 1999), in which cryoloops (see Fig. 1) are implanted into the brain to permit controlled lowering of tissue temperature to a point at which it is synaptically inactive yet viable upon rewarming. Here we designed our cryoloops to reversibly inactivate a volume of tissue $\left(\sim 162 \mathrm{~mm}^{3}\right)$ that is substantially larger that for than other reversible inactivation techniques $\left(\sim 14-33 \mathrm{~mm}^{3}\right.$; Dias and Segraves 1999; Sommer and Tehovnik 1997). The cryogenic technique also enables collection of large, repeated data sets that facilitate statistical analysis of saccadic deficits. Here we describe the effects of unilateral cryogenic inactivation of the FEF on bilateral saccadic performance in two tasks. In the step saccade task (Fig. 2A), a briefly flashed saccadic target is presented at 1 of 32 locations, allowing us to describe the saccadic deficits associated with targets distributed throughout the visual field. In addition to the expected contralateral saccadic deficits, we are also particularly interested in any ipsilateral saccadic deficits that may arise with a large but reversible lesion, given that FEF neurons with ipsilateral response fields are sparsely distributed throughout the FEF (Bruce and Goldberg 1985; Crapse and Sommer 2009; Funahashi et al. 1991; Segraves 1992). In the interleaved memory-guided and delayed saccade task (Fig. $2 B$ ), the monkeys had to first withhold a saccadic response and look to either a remembered or a persistent visual cue after offset of a central fixation point. This task permitted a direct comparison of the effects of FEF inactivation on tasks with differing requirements for spatial working memory. We are particularly interested in the preponderance of premature sac- 
A

Fig. 1. Surgical insertion of 2 cryoloops into the right arcuate sulcus. $A$ : a $5 \times 3-\mathrm{mm}$ cryoloop is positioned over the superior aspect of the arcuate sulcus before insertion. $B$ : an additional $7 \times 3-\mathrm{mm}$ cryoloop is inserted into the inferior aspect of the arcuate sulcus.

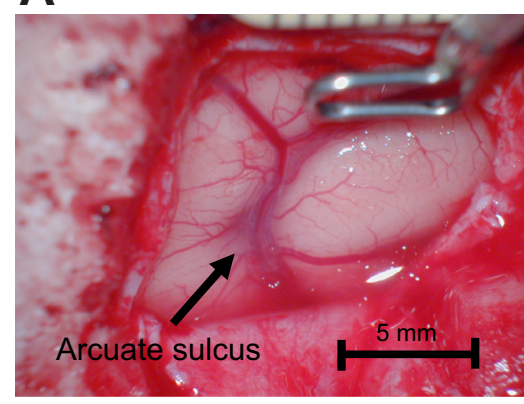

B

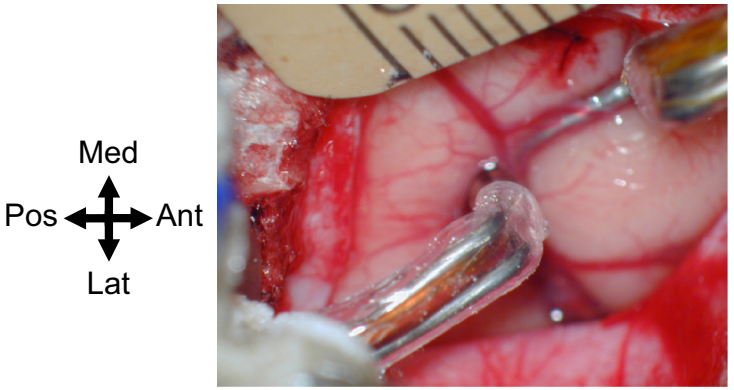

cades to ipsilateral-presented cues in this task, as such errors are prevalent after reversible pharmacological inactivation (Dias et al. 1995; Dias and Segraves 1999; Sommer and Tehovnik 1997) but not permanent ablations (Deng et al. 1986). It therefore remains unclear whether premature saccades occur only when a small volume of the FEF is inactivated, perhaps because of disinhibition of a focal, corresponding region of the noninactivated FEF (Schlag et al. 1998), or whether premature saccades are not seen after permanent ablations because of functional recovery.

Consistent with previous FEF inactivation studies, we observed the triad of contralateral saccadic deficits that usually accompany the inactivation of oculomotor structures (increased RT, decreased accuracy and peak velocity). We also found moderate, yet consistent, increases in RTs for ipsiversive saccades, even though these saccades had normal saccade accuracy and dynamics. Surprisingly, we did not observe any consistent increases in premature saccades with ipsilateral cues, which differ markedly from the substantial increases reported by reversible pharmacological inactivation studies.

Some results have been reported previously in abstract form (Peel et al. 2010).

\section{METHODS}

Subjects and physiological procedures. Two male monkeys (Macaca mulatta; monkeys $M$ and $G$, weighing 8.7 and $11.1 \mathrm{~kg}$, respectively) were used in these experiments. All training, surgical, and experimental procedures were in accordance with the Canadian Council on Animal Care policy on the use of laboratory animals (Olfert et al. 1993) and were approved by the Animal Use Subcommittee of the University of Western Ontario Council on Animal Care. The monkeys' weights were monitored daily, and their health was under the close supervision of the university veterinarians.

Each monkey underwent an aseptic procedure to implant two stainless steel cryoloops into the right arcuate sulcus as shown in Fig.
1. We customized the cryoloops based on an anatomical magnetic resonance image (MRI) obtained from each monkey, implanting in each case a $3 \times 7-\mathrm{mm}$ and a $3 \times 5$-mm (depth $\times$ length) cryoloop in the inferior and superior aspects of the arcuate sulcus [inferior arm (IA), superior arm (SA)], respectively. We performed a small 2.25$\mathrm{cm}^{2}$ craniotomy at the stereotaxic coordinates of the arcuate sulcus spur to allow for insertion of both IA and SA cryoloops. A detailed technical report of the cryoloop technique has been described previously (Lomber et al. 1999), and previous studies have implanted cryoloops in monkey cortical sulci to reversibly inactivate brain areas (Johnston et al. 2013; Nassi et al. 2013; Ponce et al. 2011). The typical drug regimen and other surgical details in the lab have been described previously (Elsley et al. 2007). In addition, monkeys were given dexamethasone postoperatively to minimize potential brain swelling.

Experimental procedures. The monkeys were placed with their heads restrained in a customized primate chair (Crist Instruments) for the duration of the experiment. We conducted experiments in a dark, sound-attenuated room, and infrared cameras were used to monitor body movements. The chair was secured at the center of a $3-\mathrm{ft}^{3}$ coil system (CNC Engineering), with the monkey facing an rectilinear grid of $>500$ red LEDs covering $\pm 35^{\circ}$ of the horizontal and vertical visual field. All aspects of the experiment were controlled by customized real-time LabVIEW programs on a PXI controller (National Instruments) operating at a rate of $1 \mathrm{kHz}$. We collected eye position signals from either a gaze-tracking coil system or a single chair-mounted eye tracker (EyeLink II) in monkeys $M$ and $G$, respectively. An experimental data set consisted of precooling (active), pericooling (inactivated), and postcooling (reactivated) sessions, with each session containing 200 or 150 correct trials $(\sim 10 \mathrm{~min})$ for monkeys $M$ and $G$, respectively. After the precooling session, we turned on the cooling pumps, initiating the flow of chilled methanol through the lumen of the cryoloops. We began the pericooling session when cryoloop temperature attained a temperature of $0-3^{\circ} \mathrm{C}$ for at least $3 \mathrm{~min}$. The temperature of the cryoloop was monitored via a wired connection from a microthermocouple to a digital thermometer. Cryoloop temperatures of $0-3^{\circ} \mathrm{C}$ silence postsynaptic activity in surrounding neurons up to $1.5 \mathrm{~mm}$ away without influencing fibers of passage
Fig. 2. Experimental saccade tasks used. $A$ : step saccade task with cues distributed throughout the visuomotor field; 32 cue locations are arranged in 8 directions with eccentricities ranging from $4^{\circ}$ to $20^{\circ}$ (gray circles represent potential cue locations). $B$ : interleaved memory and delayed saccade task to contralateral- and ipsilateral-presented cues; 5 cues are confined to the horizontal axis on both sides of the central fixation LED with eccentricities of $4-20^{\circ}$.
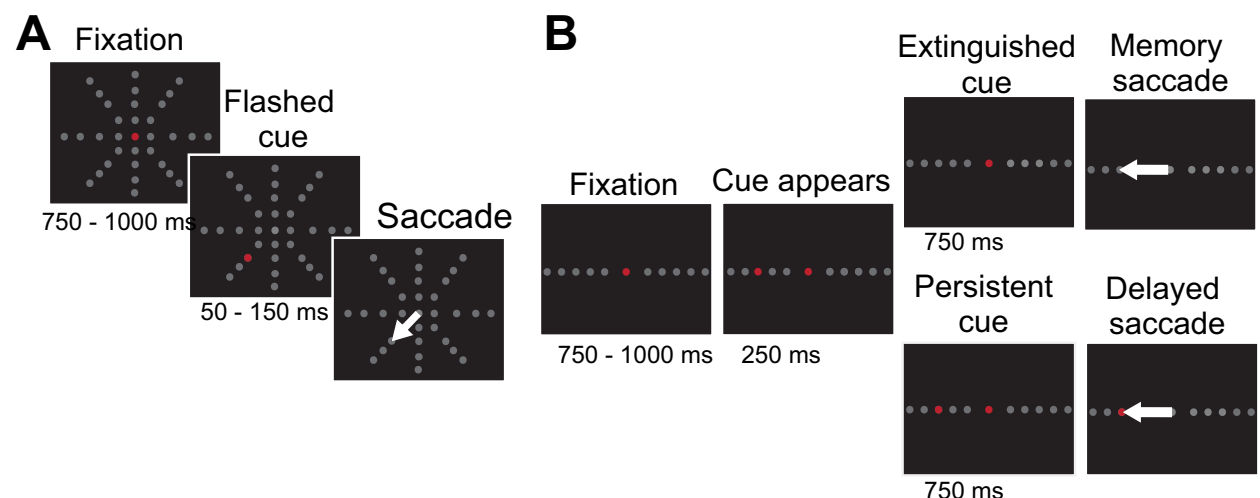

$750 \mathrm{~ms}$ 
(Lomber et al. 1999). Cooling both the IA and SA cryoloops provided an inactivation volume of $\sim 162 \mathrm{~mm}^{3}$, which is $\sim 5-10$ times larger than that in previous FEF pharmacological inactivation studies (Dias et al. 1995; Dias and Segraves 1999; Sommer and Tehovnik 1997). Note that this volume estimate does not include the volume of inactivated tissue in the posterior bank of the arcuate sulcus, as inactivation of this region does not appear to affect saccadic behavior (see DISCUSSION). Once we finished trial collection for the pericooling session, the cooling pumps were turned off, which allowed the tissue to rapidly rewarm. We commenced the postcooling session when cryoloop temperatures were within $1^{\circ} \mathrm{C}$ of temperatures observed in the precooling session $\left(\sim 37^{\circ} \mathrm{C}\right)$ for at least $3 \mathrm{~min}$. The monkeys continued to perform the behavioral task throughout the cooling and rewarming transitions, but these data are not reported here. We also conducted sham control sessions in which experimental conditions were identical to those of actual cooling days, with the exception that the flow of chilled methanol bypassed the cryoloops so that the cryoloops remained at physiological temperatures. To avoid any possible biases, we collected only one complete cooling or sham control data set per day.

Behavioral tasks. We trained monkeys to perform two behavioral tasks: a step saccade task and an interleaved memory and delayed saccade task (Fig. 2). These tasks were always performed on separate days. The step saccade task allowed us to evaluate saccades to cue locations distributed throughout the visual field. After the monkey maintained fixation of a central LED for 750-1,000 ms, a briefly flashed peripheral cue appeared simultaneously with the offset of the central LED, which signaled the monkey to generate a saccade toward the cue within $1,000 \mathrm{~ms}$. Since preliminary data showed only subtle saccadic deficits toward persistent visual cues, we flashed cues in order to increase the cognitive demands for this task, because previous research has demonstrated that greater deficits during FEF inactivation accompany more demanding tasks (Deng et al. 1986; Dias and Segraves 1999; Sommer and Tehovnik 1997). We chose flash period durations of 50 and $150 \mathrm{~ms}$ for monkeys $M$ and $G$, respectively, selecting a duration that was usually sufficient for the monkey to generate a saccade (monkeys often neglected shorter flash durations during FEF inactivation). Thirty-two possible cue locations were distributed within $\pm 20^{\circ}$ of visual angle from the central fixation LED (positive values denote right or up location). Both monkeys completed four to seven correct saccade trials to each cue location per session. These cues were arranged in eight evenly spaced directions (rotated at $0^{\circ}, 45^{\circ}, 90^{\circ} \ldots 315^{\circ}$ of straight right) at four different eccentricities $\left(4^{\circ}, 10^{\circ}, 16^{\circ}\right.$, and $\left.20^{\circ}\right)$, with the smallest amplitude varying slightly for cardinal $\left(4^{\circ}\right)$ or oblique $\left(5.7^{\circ}\right)$ directions. Acceptance windows around the target were relatively large $\left(2.8-14^{\circ}\right)$, with a diameter equal to $70 \%$ of the target's visual angle. Monkeys were required to maintain eye position within this window for $250 \mathrm{~ms}$ to be rewarded with water delivered via a sipper tube. Larger acceptance windows were necessary in this study to ensure that the monkey could be rewarded despite some degree of inaccuracy during FEF inactivation (see RESULTS).

We utilized the interleaved memory and delayed saccade task to compare the saccadic deficits toward remembered or persistent visual cues following a delay period, which enabled a more comprehensive description of various saccade errors. We also used this task to study saccade dynamics via construction of velocity-amplitude main sequence relationships. After an initial fixation period of 750-1,000 ms, a peripheral cue was presented on the horizontal axis, which either was extinguished after $250 \mathrm{~ms}$ or persisted throughout a 1,000-ms delay period where the monkey was required to maintain central fixation. The central fixation LED was then extinguished, which signaled the monkey to generate a memory or delayed saccade to either the extinguished or persistent cue location, respectively, within 1,000 ms. Both monkeys completed 15-20 correct trials to each cue location per session. Cue locations were arranged along the horizontal meridian either contralateral or ipsilateral to the central fixation LED at five different eccentricities $\left(4^{\circ}, 8^{\circ}, 12^{\circ}, 16^{\circ}\right.$, and $\left.20^{\circ}\right)$. The acceptance windows around the fixation point and cue were the same as in the step saccade task, as was the required fixation duration at the cue.

Data analysis. Eye position traces were scanned by computer algorithms in MATLAB (MathWorks) to determine the onset and offset times of saccades with a velocity criterion of $30 \%$. We analyzed the first saccade following fixation LED disappearance. Visual inspection of the data off-line by the experimenter verified whether these onset and offset marks were appropriate for saccades toward the target location and reclassified rewarded trials in which the first saccade went in the direction opposite to the cue as misdirected saccade error trials. We also discarded correct trials that had saccade end points greater than two times the target window ( $<1 \%$ of trials). Furthermore, trials with RTs $<60 \mathrm{~ms}$ were classified as premature saccade error trials.

We calculated saccade targeting error and end-point scatter for each saccade using formulae described by White and colleagues (1994). The saccade targeting error represents the mean angular distance between the displacements of cue location and individual saccade end points from the central fixation position and is defined as

$$
\mathrm{TE}_{\text {saccade }}=\sqrt{\left(X-x_{i}\right)^{2}+\left(Y-y_{i}\right)^{2}}
$$

where $X=$ horizontal displacement, in degrees, of cue location, $Y=$ vertical displacement, in degrees, of cue location, $x=$ horizontal displacement, in degrees, of saccade end point, and $y=$ vertical displacement, in degrees, of saccade end point.

Saccade end-point scatter represents the mean angular distance between the displacements of mean and individual saccade end points from the central fixation position and is defined as

$$
\mathrm{ES}_{\text {saccade }}=\sqrt{\left(\bar{x}-x_{i}\right)^{2}+\left(\bar{y}-y_{i}\right)^{2}}
$$

where $\bar{x}=$ mean horizontal displacement, in degrees, of saccade end points, $\bar{y}=$ mean vertical displacement, in degrees, of saccade end points, $x=$ horizontal displacement, in degrees, of saccade end point, and $y=$ vertical displacement, in degrees, of saccade end point.

Trials that were not successfully completed were also included in the analysis of performance errors. We observed three main error types that increased during FEF inactivation: neglect (no saccade generated), misdirected saccades (defined as saccades that were rotated $>90^{\circ}$ clockwise or counterclockwise from the appropriate saccade direction), and premature saccades (saccades initiated in any direction before or up to $60 \mathrm{~ms}$ after the fixation LED was extinguished). Since neglect or misdirected saccades error types occurred after the offset of the fixation LED, we combined these error types for statistical analyses. Our rationale for grouping these errors was also motivated by the observation that misdirected saccades generally occurred much later than correct saccades for both monkeys and tasks; therefore, both neglect and misdirected saccades error trials had a prolonged period following fixation point offset where no saccade was generated. The RTs of misdirected saccades were $144 \mathrm{~ms}$ and $100 \mathrm{~ms}$ longer than correct memory saccade trials for monkeys $M$ and $G$, respectively. These average RTs for misdirected saccades are 4.7 or 2.3 standard deviations larger than the mean RTs for correctly performed trials for monkeys $M$ and $G$, respectively, and the differences between distributions were significant (monkey $M: P<0.005$, monkey $G: P<0.05$; Wilcoxon signed-rank test).

For analyses of saccadic reaction time (SRT) and performance errors, we first collapsed data by saccade direction and then calculated statistics within each session. For analyses of saccade trajectory (e.g., targeting errors and saccade scatter) and peak velocity, we calculated the statistics on data pooled across all sessions. We compared the effects of conditions (FEF or sham cooling), tasks (when comparing delayed and memory saccades), sessions (pre-, peri-, postcooling), and cue or saccade directions (contra- and ipsilateral) on each of these saccade and performance measures. In cases where behavioral measures are compared across sessions a paired test was used with a 
Bonferroni correction for multiple comparisons (corrected $\alpha=0.05 / 2=$ 0.025), whereas repeated-measures ANOVAs were used to compare the effects of cooling across conditions, tasks, and directions $(\alpha=0.05)$. Alternatively for cases where there are multiple comparisons across cue locations, a two-sample $t$-test was used with a Bonferroni correction (corrected $\alpha=0.05 / 32=0.00156$ ). To calculate statistics for the velocity-amplitude main sequence relationship, we pooled saccades across data sets for each session and fit a nonlinear regression to independently measure changes to saccade peak velocities across sessions. To determine significant changes in saccade peak velocities independent of amplitude, we first performed a bootstrap analysis using 5,000 sets of randomly sampled saccades with replacement in a nonlinear regression fit for the function and its initial coefficients $a$ and $b$ defined as

$$
y=a\left(1-e^{\frac{-x}{b}}\right) ; a=800 ; b=35
$$

where $y=$ saccade peak velocity, in degrees per second, and $x=$ saccade amplitude, in degrees.

The nonlinear regression fit returned coefficient estimates for each of the 5,000 sets of randomly sampled saccades, which we then used to extract the peak velocities at $10^{\circ}, 15^{\circ}$, and $20^{\circ}$. Finally, we calculated the standard error and $95 \%$ confidence intervals of session distribution means and determined significant differences between distributions using Welch's $t$-tests with a Bonferroni correction for multiple comparisons (corrected $\alpha=0.025$ ).

\section{RESULTS}

Description of data set. We tested the effects of cryogenic FEF inactivation on saccadic behavior by cooling one or both of the IA and SA cryoloops. From monkey M, we collected 7 sets of pre-, peri-, and postcooling sessions of both cryoloops from the step saccade task and 10 sets from the memory and delayed saccade task. We also performed sham controls in which the cryoloops remained warm throughout time-controlled sessions and collected five and eight sets of sessions from the step or memory and delayed saccade task, respectively. From monkey $G$, we collected seven sets of cooling sessions of both cryoloops in the step saccade task, while eight sets were collected in the memory and delayed saccade task. Similarly, we also collected 7 and 12 sets of sham control sessions in the step or memory and delayed saccade task, respectively. In addition, we collected 22 and 37 sets of sessions from individual (IA or SA) cryoloop cooling from monkey $M$ and monkey $G$, respectively. For simplicity, we only report the effects of cooling both cryoloops together, since saccadic deficits were only quantitatively, and not qualitatively, less severe when individual cryoloops were cooled. Briefly, cooling only the IA cryoloop caused greater saccadic deficits than cooling the SA cryoloop alone and produced a deficit $\sim 70 \%$ as severe as that observed when both cryoloops were cooled. This estimate includes the increases in ipsiversive SRTs we observed with cooling both cryoloops (see below). Furthermore, with the exception of neglect errors, the effects of cooling both cryoloops were well predicted by adding the effects of cooling individual cryoloops alone. In contrast, neglect errors tended to be rare during cooling of individual IA or SA cryoloops but much more frequent with combined cooling of the cryoloops (see below). In sum, we found that larger FEF inactivation volumes produced larger saccadic deficits, although saccadic deficits were more apparent with a FEF inactivation in the IA compared with the SA of the arcuate sulcus. For the rest of the results, we focus on the effects of cooling both cryoloops.
Behavioral deficits profile after unilateral FEF inactivation. We observed a broad and consistent profile of saccadic deficits during unilateral FEF cryogenic inactivation on every cooling day, which at least partially recovered upon rewarming. For this report, we emphasize saccadic deficits that 1 ) were consistent in both monkeys, 2) showed some tendency for recovery upon FEF rewarming, and 3) were greater than trends observed during sham inactivation and hence could not simply be attributed to satiation or decreased motivation. Saccadic deficits included increases in bilateral-directed SRT, decreases in contraversive (leftward) saccade accuracy, peak velocity, and amplitude, and increases in several performance errors (neglect, misdirected saccades). We first describe saccadic deficits toward flashed cues distributed throughout the visual field in the step saccade task and then describe the changes in saccade behavior in the interleaved memory and delayed saccade task. Overall, both monkeys continued to perform well during FEF inactivation, even though performance errors increased. Additionally, we did not see any increases in the proportion of missed trials during FEF inactivation (e.g., where the monkey failed to initiate the trial by not looking at the central fixation LED). During FEF inactivation, we also did not observe any substantial $\left(>1^{\circ}\right)$ changes in fixation eye position or propensity for erroneous saccades in the fixation interval preceding cue presentation. We also did not detect any abnormalities in monkeys' nonsaccadic behavior during FEF inactivation, although we acknowledge that we did not specifically test limb or hand movements. Indeed, nonsaccadic deficits are likely since the inactivation volume extended to the posterior bank of the arcuate sulcus, encompassing functional areas in the premotor cortex related to coordinated visually guided arm and hand movements (Halsband and Passingham 1982; Moll and Kuypers 1977; Weinrich et al. 1984; Weinrich and Wise 1982).

Unilateral FEF inactivation increased targeting errors for contraversive saccades. We first describe saccade trajectories and errors to flashed cue locations distributed throughout the visual field after unilateral inactivation of the right FEF. Before FEF inactivation, both monkeys could generate accurate saccades to all briefly flashed cue locations (Fig. 3, $A$ and $B$ ). Saccade amplitudes scaled with cue eccentricity, although monkey $G$ 's rightward (ipsilateral) saccades showed substantial hypometria (Fig. 3B). Recall that this figure only shows the first saccade; we confirmed that monkey $G$ attained all flashed cues with a subsequent saccade(s). Monkey $G$ also generated normometric saccades to persistent visual targets (see Fig. 9B, bottom). In both monkeys, FEF inactivation increased hypometria and end-point scatter for saccades toward contralateral cues (Fig. 3, $C$ and D; these changes are difficult to resolve for monkey $M$ given the scaling but are analyzed quantitatively in the next section). Hypometria tended to be greatest for more eccentric contralateral cues, whereas less eccentric cues primarily exhibited increased end-point scatter. Monkey $G$ displayed quantitatively larger deficits with more pronounced hypometria to contralateral cue locations, and to upward and downward locations as well. Upon rewarming, both monkeys showed considerable recovery in saccade amplitude and endpoint scatter, particularly for those cues most affected by FEF inactivation (Fig. 3, $E$ and $F$ ). In summary, both monkeys had increased targeting errors for contralateral cues during FEF inactivation characterized by hypometria and increased end- 
Trajectories (cooling)
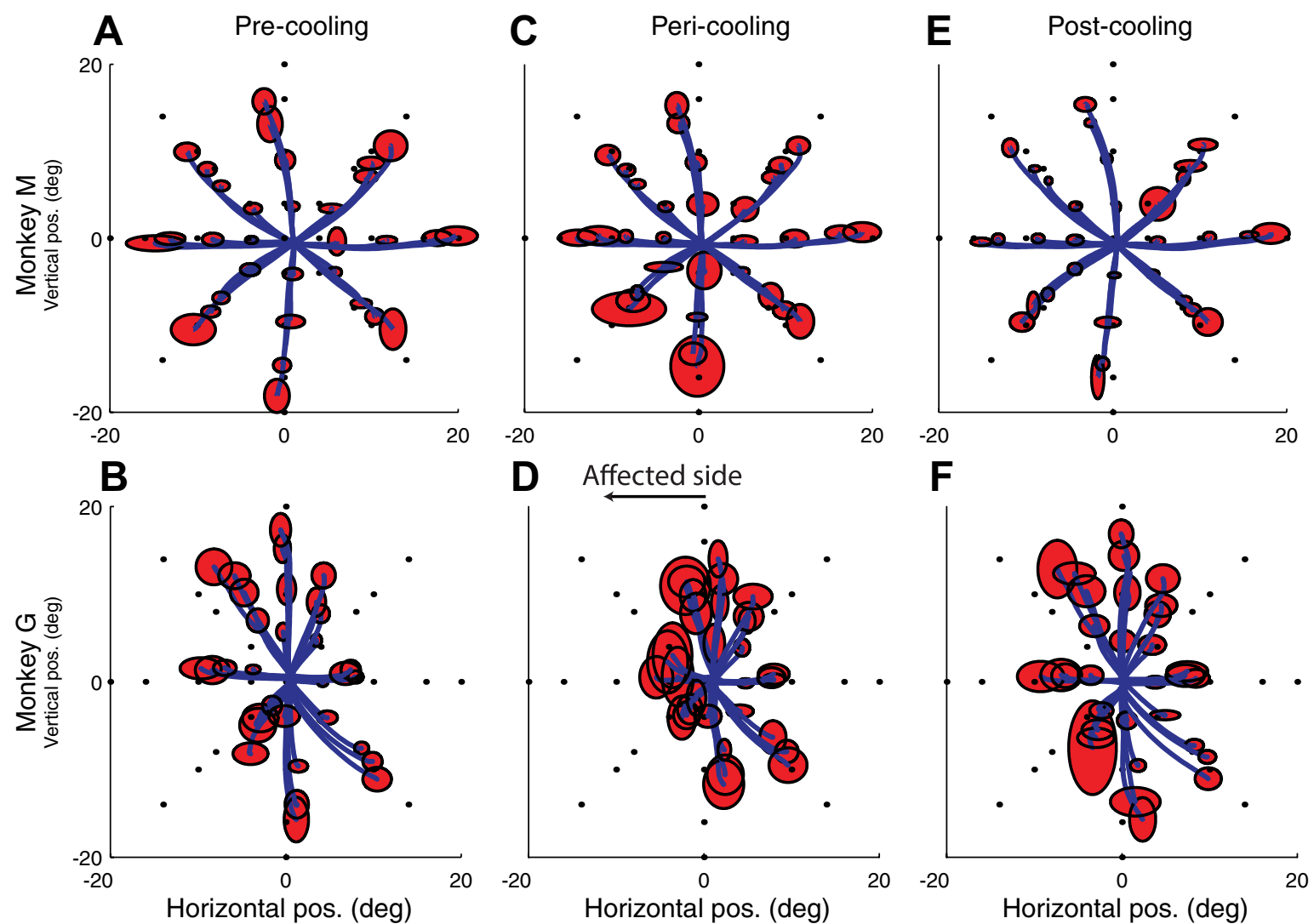

Fig. 3. Saccade trajectories and end points to briefly flashed cues distributed throughout the visual field before $(A$ and $B)$, during $(C$ and $D)$, and after $(E$ and $F$ ) unilateral frontal eye field (FEF) inactivation for each monkey. Mean saccade trajectory (blue curve) was calculated from individual trajectories pooled across days for each session. Red ellipses represent $\pm 1 \mathrm{SD}$ of the horizontal and vertical saccade end-point scatter.

point scatter toward flashed contralateral cues, which showed substantial recovery upon FEF rewarming.

Quantitative comparison of saccade targeting error across all cue locations. To quantify and compare these trajectory deficits across all cue locations and to sham control sessions, we constructed contour plots representing the change in targeting error for each cue location. The change in saccade targeting error is computed for the cooling and warming transitions as

$$
\begin{aligned}
& \text { Change across cooling transition : } \overline{\mathrm{TE}}_{\mathrm{during}} / \overline{\mathrm{TE}}_{\mathrm{before}} \\
& \text { Change across rewarming transition : } \overline{\mathrm{TE}}_{\mathrm{after}} / \overline{\mathrm{TE}}_{\text {during }}
\end{aligned}
$$

Since increases in targeting errors following transitions produce ratios $>1$, larger values represent situations in which saccades become more inaccurate across the transition. We observed significant increases in the targeting error for $33 \%$ (4/12) and 92\% (11/12) of contralateral cues for monkey $M$ (Fig. 4A) and monkey $G$ (Fig. 4B), respectively (2-sample $t$-tests, Bonferroni corrected for multiple comparisons; this analysis excludes saccades to purely vertical cues). Targeting error ratio increases mainly arose from saccade hypometria and could increase by a value of 1.5 or more, particularly for contralateral cues. Both monkeys exhibited increased targeting error ratios for contralateral, downward, and select ipsilateral cues. A trend for an increasing degree of hypometria for more eccentric cues was also apparent in both monkeys. Statistically significant hypometria was observed for cue eccentricities $>$ $12^{\circ}$ in $38 \%(6 / 16)$ and $63 \%(10 / 16)$ cases for monkeys $M$ and $G$, respectively, whereas hypometria only reached significance for cue eccentricities $<12^{\circ}$ in $13 \%(2 / 16)$ and $44 \%(7 / 16)$ cases for monkeys $M$ and $G$, respectively. Upon FEF rewarming, targeting error ratios recovered to some degree in both monkeys, especially for those cue locations most affected during FEF inactivation (Fig. 4, $C$ and $D$ ). Such recovery reached significance only in monkey $G$ for 14 of the 18 cue locations significantly affected by FEF inactivation. In contrast to the changes in the targeting error ratio seen with actual cooling and rewarming of the FEF, sham inactivation produced only minimal and largely nonsignificant changes in the targeting error ratio for each monkey across any transition (Fig. 4, $E-H)$. Thus the increases in hypometria for contraversive saccades during FEF inactivation are not simply attributable to satiation or decreased motivation.

We next investigated the changes in saccade end-point scatter across cue locations, by constructing contour plots using saccade end-point scatter ratio for each monkey in a similar manner. The change in saccade end-point scatter is computed for the cooling and warming transitions as

$$
\begin{aligned}
& \text { Change across cooling transition : } \overline{\mathrm{ES}}_{\text {during }} / \overline{\mathrm{ES}}_{\text {before }} \\
& \text { Change across rewarming transition }: \overline{\mathrm{ES}}_{\mathrm{after}} / \overline{\mathrm{ES}}_{\text {during }}
\end{aligned}
$$

Since increases in end-point scatter following transitions produce ratios $>1$, larger values represent situations in which 

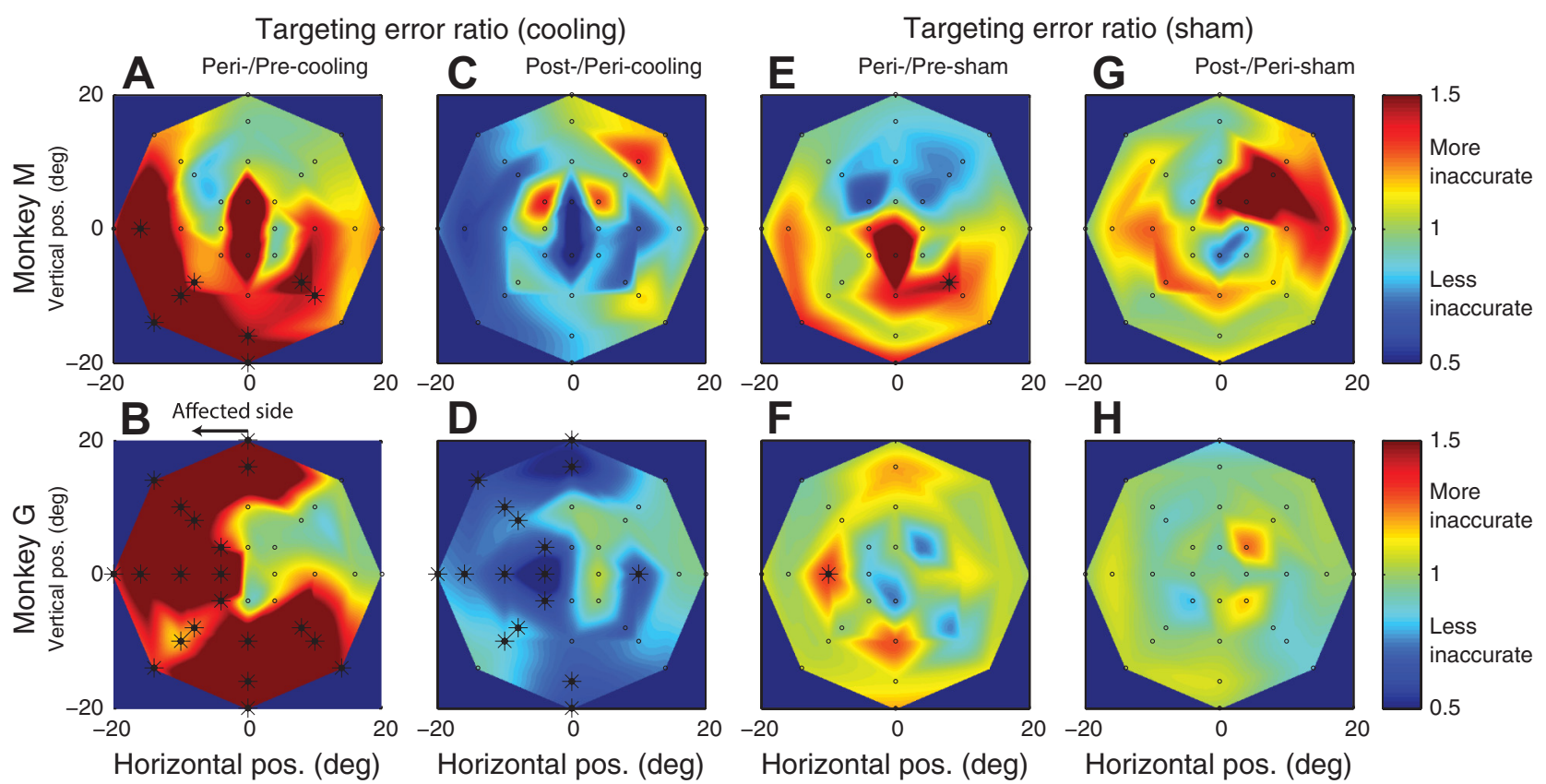

Fig. 4. Relative change of saccade targeting error to briefly flashed cues distributed throughout the visual field after unilateral FEF inactivation for monkey $M$ $(A)$ and monkey $G(B)$ and upon FEF rewarming for monkey $M(C)$ and monkey $G(D)$. For comparison, the relative change of saccade targeting error is shown after a sham inactivation for monkey $M(E)$ and monkey $G(F)$ and upon reversal for monkey $M(G)$ and monkey $G(H)$. The targeting error ratio was calculated for each of the 32 target locations across cooling days. Increases in saccade targeting error ratio are indicated by red tints, decreases by blue tints, and negligible changes by green tints. Significant changes in saccade targeting error ratio are represented by asterisks for each target location, using a paired $t$-test with a Bonferroni correction $(P<0.00156)$.

saccades become more variable across the transition. After FEF inactivation, we observed increases of the saccade endpoint scatter ratio exceeding 1.5 to several contralateral cue locations, including some of those that also had concomitant large increases in targeting error ratio (Fig. 5, $A$ and $B$ ). Significant increases in the saccade end-point scatter ratio were observed for $17 \%(2 / 12)$ and $42 \%(5 / 12)$ of contralateral cues for monkeys $M$ and $G$, respectively (paired $t$-tests, Bonferroni corrected for multiple comparisons; excluding vertical sac- cades). While monkey $M$ exhibited a similar spatial profile in the changes of the targeting error ratio and saccade end-point scatter ratio, for monkey $G$ significant increases in saccade end-point scatter tended to be restricted to less eccentric contralateral cues, unlike the spatial profile observed for targeting error ratio. This result may be related to the larger degree of hypometria exhibited by this monkey or the greater propensity for this monkey to neglect eccentric contralateral cues (see below). Upon rewarming, the changes in saccade
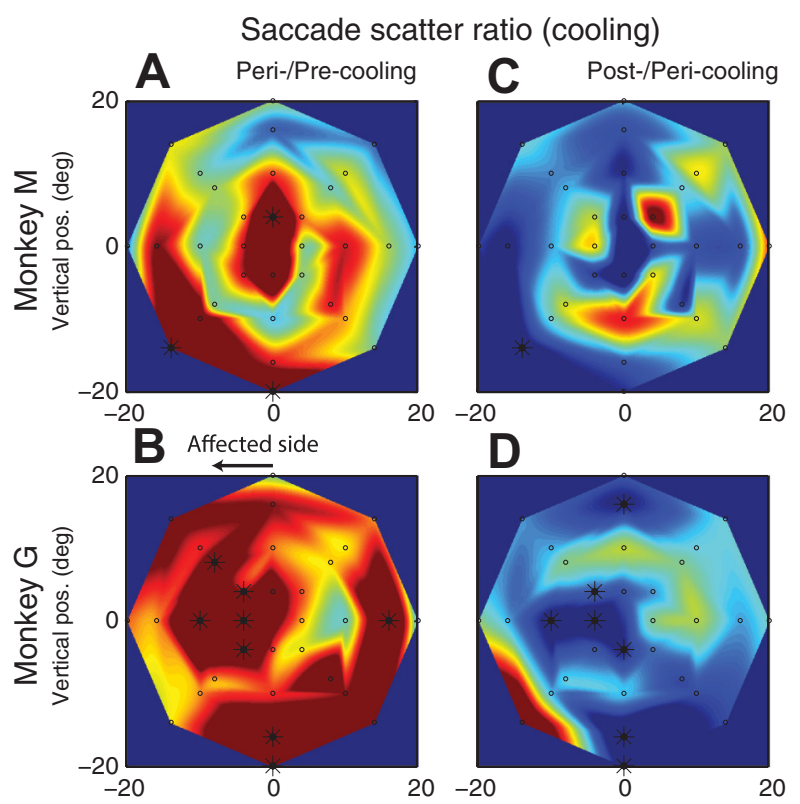

Horizontal pos. (deg) Horizontal pos. (deg)

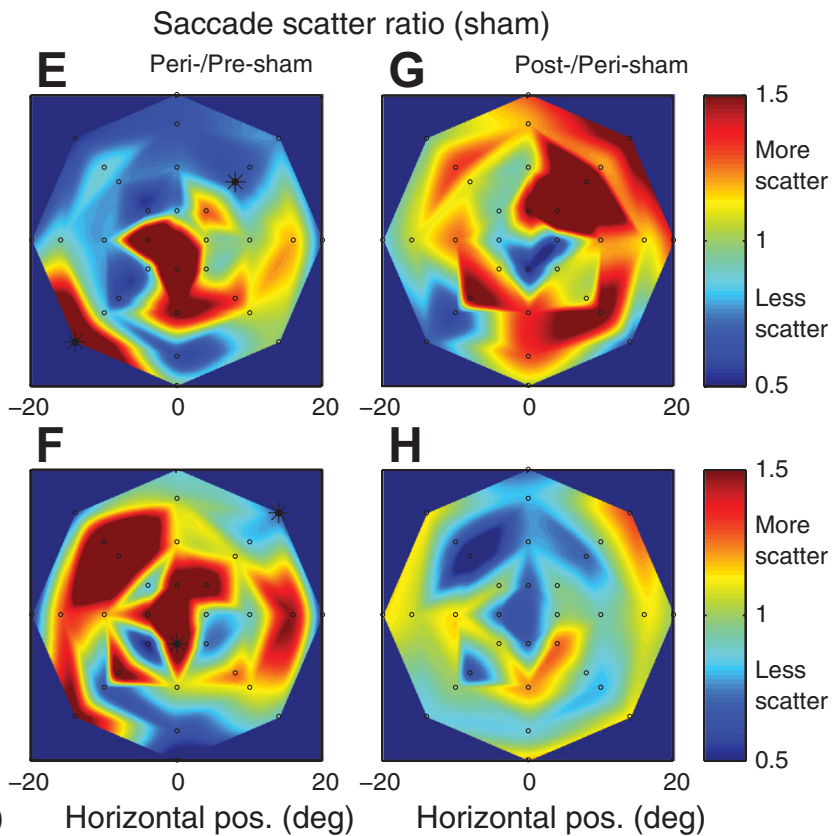

Fig. 5. Relative change of saccade scatter to briefly flashed cues distributed throughout the visual field with unilateral FEF inactivation. Same format as Fig. 4. 
end-point scatter generally recovered, primarily for those cue locations most affected during FEF inactivation (Fig. 5, $C$ and $D)$, with significant recovery of end-point scatter being observed in monkey $G$ at five of the eight cue locations significantly affected by FEF inactivation. Again, sham inactivation produced little consistent effect on saccade scatter across any transition (Fig. 5, $E-H$ ).

In summary, unilateral FEF inactivation in both monkeys increased hypometria and end-point scatter to almost all contralateral cues and to some ipsilateral cues close to the vertical midline. In both monkeys, these deficits tended to be more severe for more eccentric cues.

Unilateral FEF inactivation increases SRTs toward flashed cues bilaterally. We next evaluated the changes in SRTs following unilateral FEF inactivation. First, we represent the SRTs pooled across contralateral or ipsilateral locations (Fig. 6; excluding vertical cues). During FEF inactivation, both contralateral and ipsiversive SRTs significantly increased for monkey $M$ (contra $P<0.0001$, ipsi $P<0.05$ ) and monkey $G$ (contra $P<0.0001$, ipsi $P<0.01$, paired $t$-tests, Bonferroni corrected for multiple comparisons), with greater SRT increases accompanying contralateral versus ipsilateral cues (SRTs increased to contralateral cues by 54 and $134 \mathrm{~ms}$ for monkeys $M$ and $G$, respectively, and for ipsilateral cues by 44 and $21 \mathrm{~ms}$ for monkeys $M$ and $G$, respectively). Upon rewarming, bilateral-directed SRTs deficits showed some recovery, although only the SRT decrease for monkey $G$ 's contraversive saccades reached significance $(P<0.0001)$. The absence of consistent SRT recovery may be partly due to the monkeys' satiation, since SRTs also increased during sham inactivation and again upon reversal. However, the increase in SRT seen during FEF inactivation was far greater than that observed during sham inactivation, as revealed by a two-way ANOVA for both monkeys using only data from the FEF and sham inactivation session. For monkey $M$ during FEF/sham inactivation, we found significant effects for saccade directions (contra- and ipsilateral; $P<0.0001$ ) and conditions (cooling and sham; $P<0.0001$ ) on SRTs. A similar ANOVA analysis for monkey $G$ during FEF/sham inactivation revealed significant effects for saccade directions $(P<0.0001)$ and conditions $(P<0.0001)$ on SRTs. We also found a significant two-way interaction of saccade direction and condition $(P<0.0001)$. In general, we found bilateral-directed SRT increases for both monkeys during FEF inactivation, with greater increases accompanying contraversive saccades.
Quantitative comparison of SRT changes across all cue locations. We also investigated the changes in SRT at each cue location by constructing contour plots of the change in SRTs across cooling and rewarming (Fig. 7). After FEF inactivation, we observed SRT increases at most cue locations, with the largest increases accompanying contraversive saccades (Fig. 7, $A$ and $B$ ). Such SRT increases reached significance for $50 \%$ $(6 / 12)$ and $100 \%$ (12/12) of contralateral cue locations for monkeys $M$ and $G$, respectively (2-sample $t$-tests, Bonferroni corrected for multiple comparisons; excluding vertical cues). SRTs toward these cue locations increased in the range of 50-100 and 50-200 ms for monkeys $M$ and $G$, respectively. For monkey $M$, SRT increases tended to be greatest for more eccentric and upward contralateral cues. For monkey $G$, SRT increases tended to be greatest for downward contralateral cues. Upon FEF rewarming, SRTs for both monkeys tended to either recover or remain stable (Fig. 7, $C$ and $D$ ). Such recovery only reached significance in monkey $G$, doing so in $76 \%(13 / 17)$ of the cases where significant SRT increases were seen with FEF inactivation. In contrast to the large and spatially contiguous SRT changes with FEF inactivation, we observed mostly patchy and insignificant changes in SRTs during sham inactivation (Fig. 7, $E-H$ ), which we attribute to the effects of satiation or decreased motivation. In summary, SRTs increased for both monkeys during FEF inactivation, particularly for contralateral locations.

Increased neglect and misdirected saccades for flashed contralateral cues during unilateral FEF inactivation. During FEF inactivation, two types of errors were commonly observed in the step saccade task. Monkeys often neglected to look at a flashed cue (a neglect error) or looked in the opposite direction (a misdirected saccade error). Before FEF inactivation, both monkeys had low levels of combined neglect and misdirected saccade errors $(<5 \%$; Fig. 8). During unilateral FEF inactivation, monkey $G$ displayed a marked increase in the tendency to either neglect contralateral cues or look in the opposite direction (Fig. $8 B ; P<0.025$, paired $t$-tests, Bonferroni corrected for multiple comparisons). In contrast, monkey $M$ had a mild, yet significant increase in errors toward ipsilateral cues (Fig. $8 A ; P<0.025)$. After FEF rewarming, monkey $G$ 's error rate substantially recovered, nearly reaching significance $(P=$ 0.05 ), while monkey $M$ 's error rate increased, possibly because of satiation, as similar increases were seen in the sham condition. A two-way ANOVA for monkey $G$ during FEF/sham inactivation revealed significant effects of cue directions (con-

\section{Saccade reaction times}
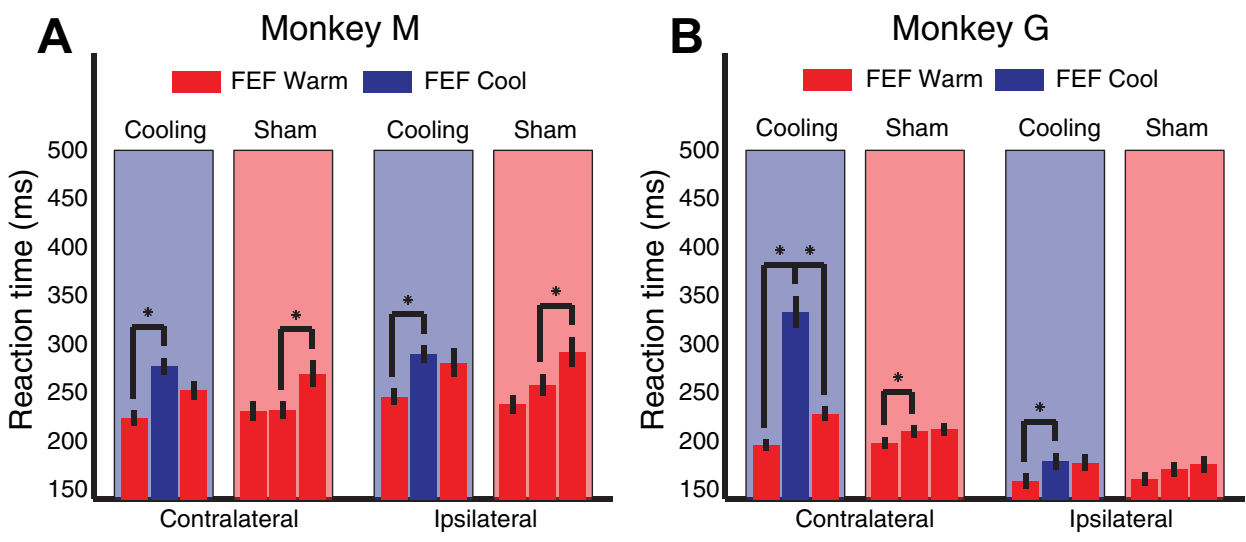

Fig. 6. Comparison of saccade reaction times (SRTs) (means $\pm \mathrm{SE}$ ) toward contralateral and ipsilateral briefly flashed cues for cooling (blue shading) and sham (red shading) conditions before, during, and after unilateral FEF inactivation. Sessions where the FEF is active or inactivated are shown by red or blue, respectively. Significant differences across transitions are indicated by asterisks if significance was reached by a $t$-test with a Bonferroni correction $(P<0.025)$. 

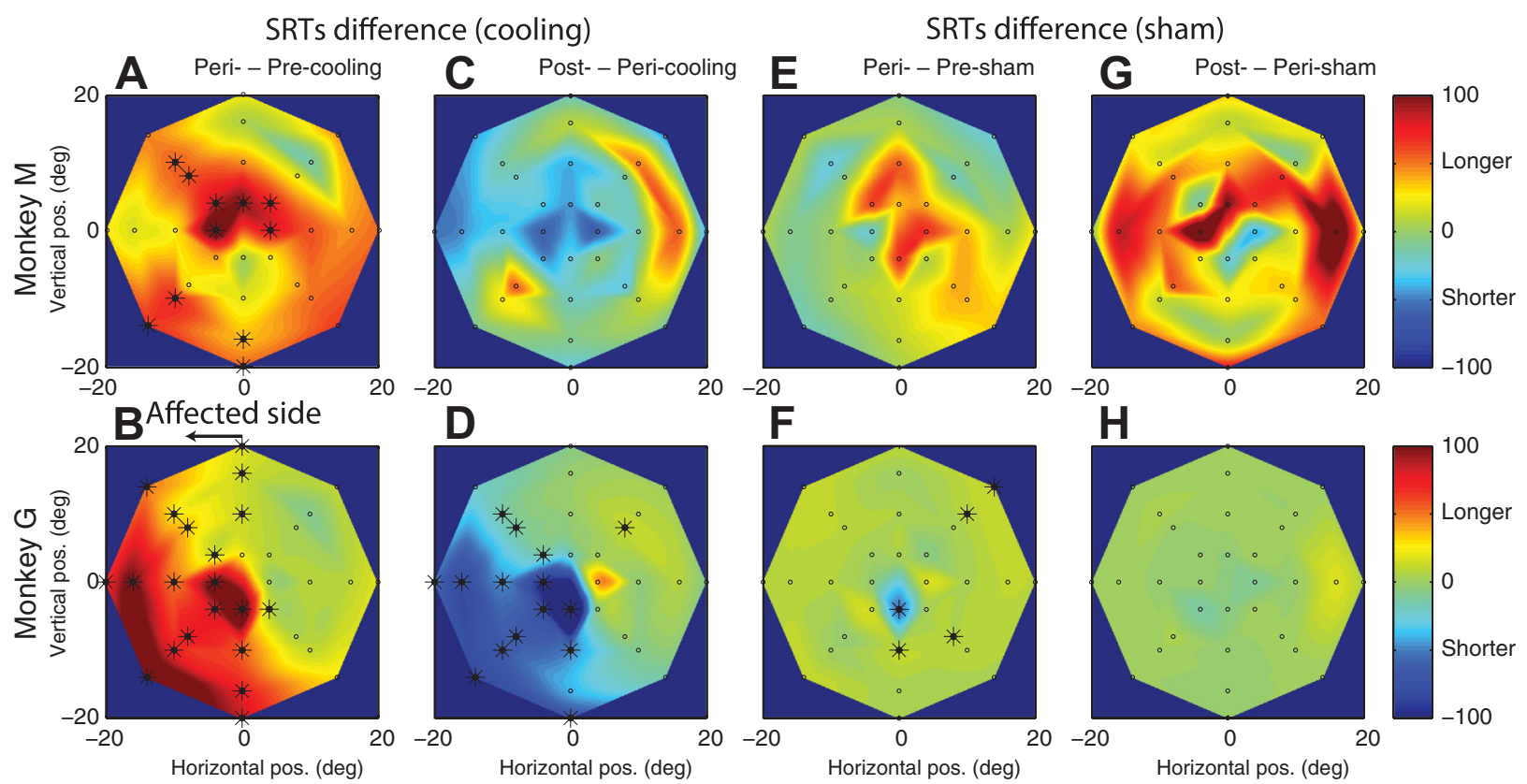

Fig. 7. Absolute difference of SRTs to briefly flashed cues distributed throughout the visual field with an unilateral FEF inactivation. Same format as Fig. 4.

tra- and ipsilateral; $P<0.01$ ) and conditions (cooling and sham; $P<0.01$ ) on combined neglect and misdirected saccade error rate. We also found a significant two-way interaction of cue direction and condition $(P<0.005)$. Using a similar ANOVA analysis for monkey $M$ during FEF/sham inactivation, we found no significant effects of factors on error rate or significant interactions of factors.

Summary of saccadic deficits for step saccade task. We used the step saccade task during unilateral FEF inactivation to quantify saccadic deficits toward flashed cue locations across the visual field. Unilateral FEF inactivation increased saccade targeting errors, end-point scatter, RTs, and in one monkey neglect and misdirected saccades. In general, we found greater impairments toward contralateral cue locations than ipsilateral locations; however, moderate deficits were observed for oblique ipsiversive saccades. We also found greater increases of saccadic deficits for saccades toward eccentric cue locations throughout the visual field compared with near locations. Both monkeys presented similar saccadic deficit profile during FEF inactivation, but the severity of saccadic deficits was greater in monkey $G$ than monkey $M$. Monkey $G$ also displayed a greater propensity for neglect and misdirected saccade errors. Although data are not shown for saccade peak velocity and amplitude relationship for this task, we did find decreases in both monkeys' saccade peak velocity independent of its amplitude during FEF inactivation for contraversive saccades only.

Memory saccades showed greater increases in targeting errors and end-point scatter than delayed saccades during FEF inactivation. We now describe changes in saccadic behavior with unilateral FEF inactivation in a task that requires a delayed response to either a remembered (memory saccades) or persistent (delayed saccades) visual target. Here we compared the effects of FEF inactivation on saccade trajectories and end points between memory and delayed saccades. In the precooling session, both monkeys displayed greater hypometria toward remembered cue locations (Fig. 9, $A$ and $B$ ), although monkey $G$ had relatively greater hypometria toward rightward than leftward targets. We attribute this rightward hypometria to an idiosyncrasy in his normal behavior since he could generate
Fig. 8. Comparison of neglect or misdirected saccade errors across contralateral and ipsilateral briefly flashed cues for cooling (blue shading) and sham (red shading) conditions before, during, and after an unilateral FEF inactivation. Same format as Fig. 6. Values represent the proportion of error trials divided by total trials in a session (mean $\pm \mathrm{SE}$ ratio). Proportions of neglect errors are represented by red and dark blue with an active (FEF warm) and inactivated FEF (FEF cool), respectively. Misdirected saccade errors are represented by orange and light blue with an active and inactivated FEF, respectively. Significant differences within both loop and sham cooling groups are indicated by asterisks if significance is reached by a $t$-test with a Bonferroni correction $(P<0.025)$.

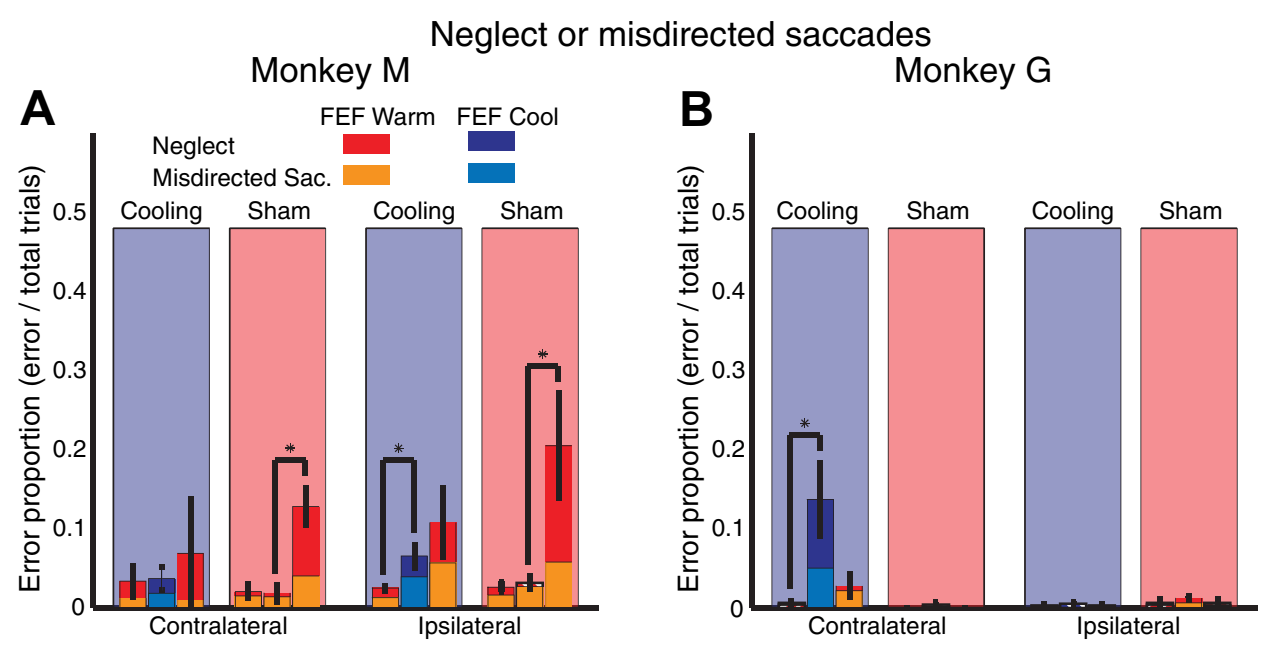

J Neurophysiol $•$ doi:10.1152/jn.00398.2013 • www.jn.org 


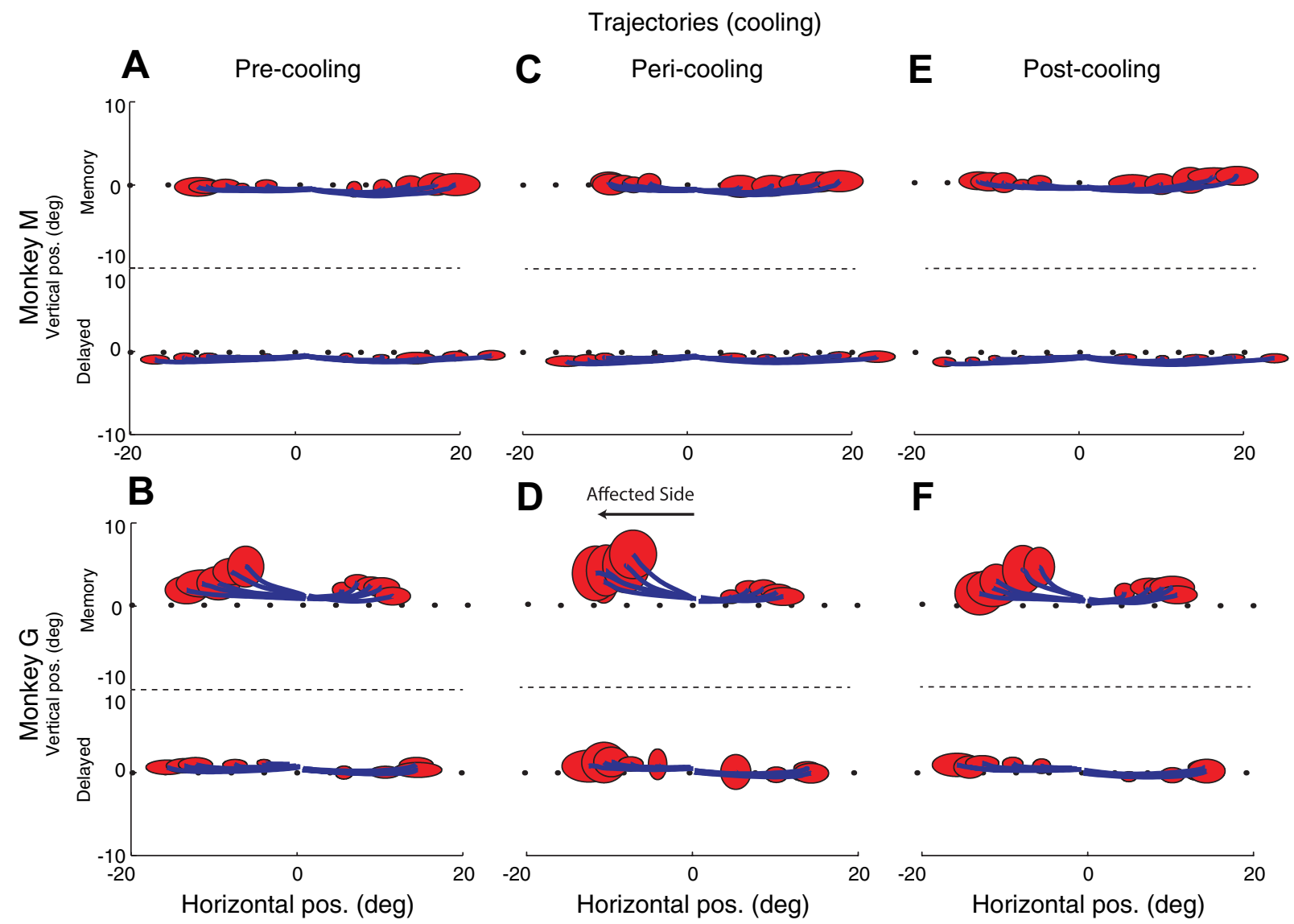

Fig. 9. Trajectories and end points of memory (top) and delayed (bottom) saccades. Same format as Fig. 3.

normometric delayed saccades (Fig. 9B, bottom). During FEF inactivation, memory and delayed saccades from both monkeys showed increased hypometria and end-point scatter, primarily for contralateral cue locations (Fig. 9, $C$ and $D$ ). In addition, for both monkeys, we found greater targeting errors for memory than delayed saccades and saccade targeting errors were more severe for peripheral, contralateral cue locations than cues near the central fixation target. During FEF inactivation, we also found mild increases in end-point scatter for both monkeys' ipsiversive saccades, particularly for saccades directed toward cue locations near the central fixation target. To determine the quantitative differences in saccade targeting error and end-point scatter following FEF inactivation, we calculated the ratio of error values across the cooling transition for each of the five contralateral and ipsilateral cue locations. In both monkeys and tasks, we found significant increases in target error ratios primarily toward contralateral cue locations. Specifically, for memory saccades, we found significant increases of $\sim 1.5$ at four of five and two of five contralateral cue locations for monkeys $M$ and $G$, respectively. For delayed saccades, we found significant increases of $\sim 1.6$ at three of five contralateral cue locations for both monkeys. Similarly, we found increases in end-point scatter primarily toward contralateral cue locations, which reached significance only for memory saccades in both monkeys (ratios increased by $\sim 1.3$, reaching significance for 2 of 5 cue locations). Upon rewarming, for both monkeys, saccade targeting error and end-point scatter errors decreased, and their saccades had metrics comparable to the precooling session (Fig. 9, $E$ and $F$ ).
Unilateral FEF inactivation preferentially impaired SRTS toward remembered cues bilaterally. Next, we describe the changes in SRT in this task, measured from the time of the offset of the central fixation LED. Unilateral FEF inactivation produced greater increases in contraversive SRTs toward remembered compared with persistent visual cue locations for both monkeys (Fig. 10), increasing for monkey $M$ by 106 and $44 \mathrm{~ms}$ for memory and delayed saccades, respectively (memory and delay both $P<0.0001$, paired $t$-tests, Bonferroni corrected for multiple comparisons), and for monkey $G$ by 64 and $52 \mathrm{~ms}$, for memory and delayed saccades, respectively (memory and delayed both $P<0.0001$ ). We also observed significant SRT increases for ipsiversive saccades, particularly for memory saccades, although these increases were smaller than that observed for contraversive saccades. For monkey $M$ ipsiversive SRTs significantly increased by 65 and $25 \mathrm{~ms}$ for memory and delayed saccades, respectively (both $P<0.0001$ ), and for monkey $G$ ipsiversive SRTs significantly increased by 32 and $29 \mathrm{~ms}$ for memory or delayed saccades, respectively (memory $P<0.01$, delayed $P<0.0001$ ). To determine significant effects and interactions of cooling conditions (cooling and sham), tasks (memory and delayed saccades), and saccade direction (contra- and ipsilateral) on SRTs, we used a three-way ANOVA using data only during FEF/sham inactivation. For monkey $M$, we found significant effects of saccade conditions $(P<0.0001)$ and tasks $(P<0.0001)$ but no significant effects of directions on SRTs. We also found significant two-way interactions of condition and task $(P<$ $0.001)$ and saccade direction and condition $(P<0.001)$. A 
Fig. 10. Comparison of SRTs (mean \pm SE) of memory and delayed saccades for cooling (blue shading) and sham (red shading) conditions before, during, and after an unilateral FEF inactivation. Contraversive saccades for monkey $M(A)$ and monkey $G(B)$ and ipsiversive saccades for monkey $M(C)$ and monkey $G(D)$ are shown. Same format as Fig. 6.

\section{Saccade reaction times}
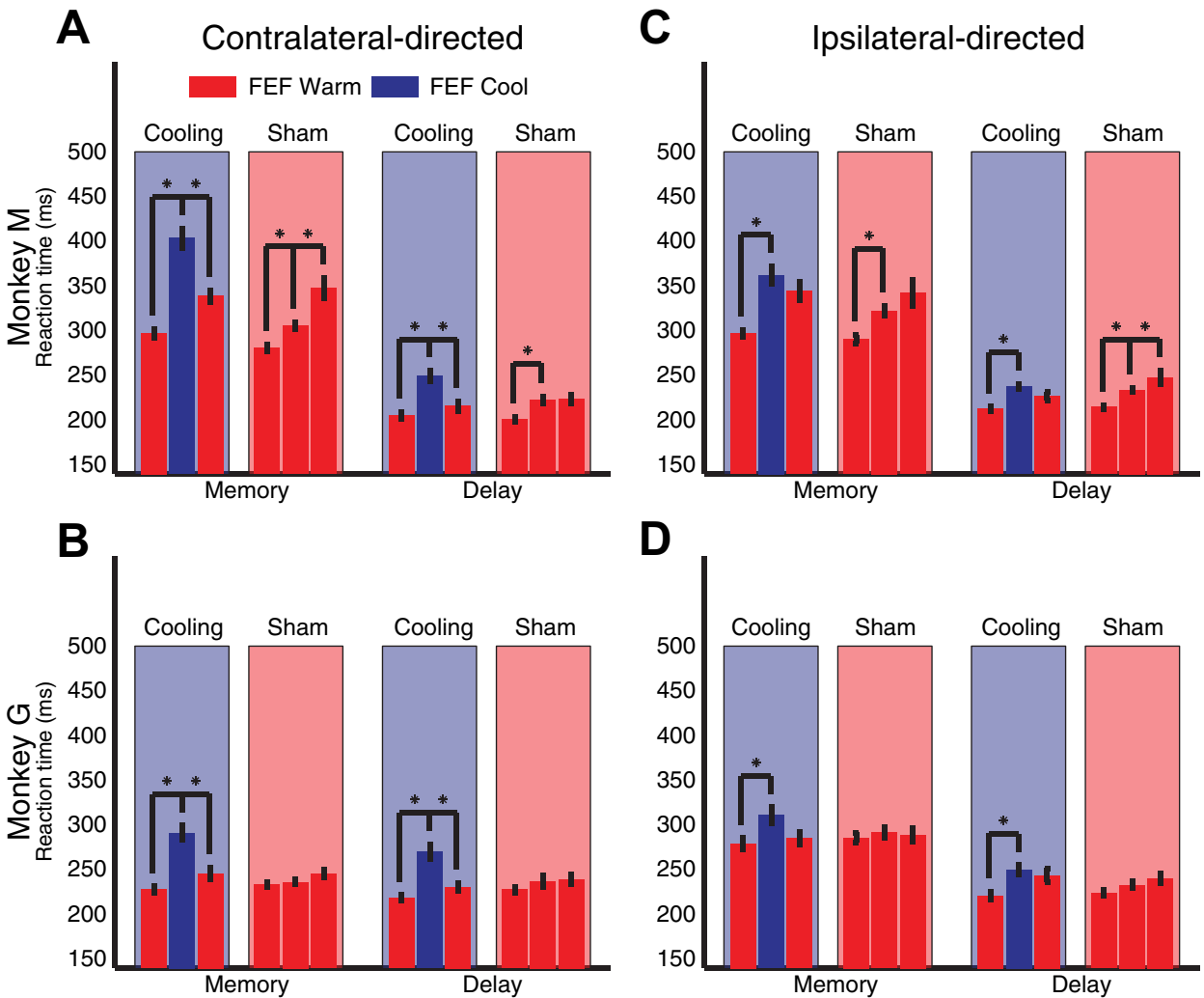

similar ANOVA analysis for monkey $G$ during FEF inactivation revealed significant effects for tasks $(P<0.0001)$ and cooling conditions $(P<0.001)$ on SRTs. We found a significant two-way interaction of saccade direction and condition $(P<0.05)$. Upon FEF rewarming, SRTs for both monkeys partly recovered to similar levels observed in the sham inactivation postsession. In summary, both monkeys presented greater increases in bilateral-directed SRTs for memory versus delayed saccades, with the greatest increases in SRTs accompanying contraversive saccades.

Unilateral FEF inactivation slowed all saccades toward contralateral cues. We also studied the velocity-amplitude main sequence and compared any changes with FEF inactivation across task and direction. During FEF inactivation, we observed substantial downward shifts in a nonlinear function fit to the velocity-amplitude main sequence relationships for contraversive memory-guided saccades that recovered upon rewarming (Fig. 11, $A$ and $B$ ). Importantly, this downward shift did not depend on any accompanying hypometria, as changes in peak velocity are evident even for saccades of moderate amplitude (e.g., $10^{\circ}$ ). To determine significant differences in peak velocities with FEF inactivation, we performed a bootstrap analysis of a nonlinear regression model, which we used to extract the peak velocities at $10^{\circ}, 15^{\circ}$, and $20^{\circ}$ in amplitude. For saccade peak velocities extracted at $10^{\circ}$ or $15^{\circ}$ in amplitude, we found significant peak velocity decreases of contraversive saccades during FEF inactivation for both monkey $M$ $\left(10^{\circ}\right.$ and $15^{\circ}$, both $\left.P<0.001\right)$ and monkey $G\left(10^{\circ}, P<0.001\right.$; $\left.15^{\circ}, P<0.01\right)$. In addition, FEF inactivation resulted in significant decreases in peak velocities extracted at $20^{\circ}$ in amplitude for only monkey $M(P<0.001)$. In contrast, no significant changes were found for ipsiversive saccades for both monkeys using extracted peak velocities at $10^{\circ}, 15^{\circ}$, and $20^{\circ}$ in amplitude (Fig. 11, $C$ and $D$ ). Upon rewarming, peak velocities of contraversive saccades extracted at $10^{\circ}, 15^{\circ}$, or $20^{\circ}$ in amplitude significantly increased in both monkey $M$ $\left(10^{\circ}, 15^{\circ}\right.$, and $20^{\circ}$, all $\left.P<0.001\right)$ and monkey $G\left(10^{\circ}\right.$ and $15^{\circ}$, both $\left.P<0.001 ; 20^{\circ}, P<0.01\right)$. Furthermore, we also found significant downward shifts in the saccade peak velocityamplitude main sequence relationship for both monkeys' contraversive delayed saccades at $10^{\circ}, 15^{\circ}$, and $20^{\circ}$ in amplitude (Fig. 12, $A$ and $B$; all $P<0.001$ ), which also recovered upon rewarming $(P<0.001)$. In contrast, FEF inactivation did not significantly influence the main sequence relationships for ipsiversive delayed saccades (Fig. 12, $C$ and $D$ ). Thus, in contrast to the bilateral effects of FEF inactivation on SRTs, the effects of FEF inactivation of velocity-amplitude relationships are unilateral, selectively shifting the relationship down for contraversive memory or delayed saccades.

Preferential increase in neglect and misdirected saccades during unilateral FEF inactivation for contraversive memory saccades. For a variety of error classes, we computed the frequency of errors before, during, and after unilateral FEF inactivation, beginning first with neglect and misdirected saccades. During unilateral FEF inactivation, both monkeys frequently either neglected to look to a remembered contralateral cue or looked in the wrong direction after disappearance of the central fixation LED (Fig. 13, $A$ and $B$ ). Since both neglect and misdirected saccades errors occurred after offset of central fixation LED and were significantly delayed compared with SRTs for correct trials (see METHODS), we pooled them together for statistical analysis. We found that both monkeys had 

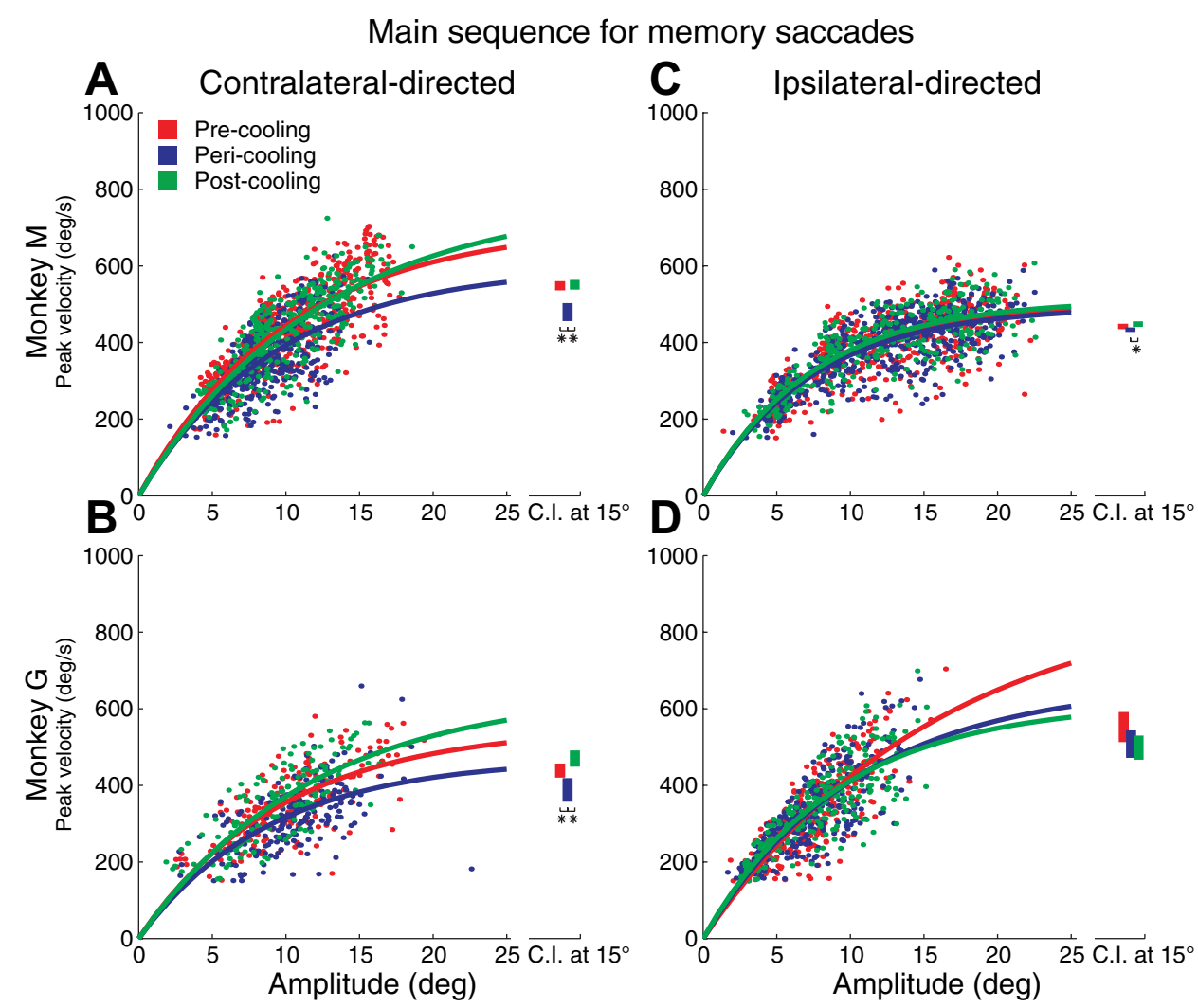

Fig. 11. Peak velocity-amplitude main sequence relationship for memory-guided saccades to contralateral and ipsilateral targets before (red lines and symbols), during (blue), and after (green) unilateral FEF inactivation. Contraversive saccades are shown first for monkey $M(A)$ and monkey $G(B)$ and ipsiversive saccades for monkey $M(C)$ and monkey $G(D)$. For each session, an exponential regression function fitted the main sequence relationship, and its confidence interval (C.I.) at $15^{\circ}$ is shown on right with a bootstrap analysis. Significant differences in peak velocity at $15^{\circ}$ across transitions are shown by asterisks, which are located below the confidence intervals.

significant increases in error frequency during FEF inactivation in memory saccade trials, using paired $t$-tests, Bonferroni corrected for multiple comparisons (monkey $M P<0.005$, monkey $G P<0.01)$. In contrast, we observed less dramatic increases in neglect and misdirected saccade errors for delayed saccades to persistent visual, contralateral cues, which approached significance for both monkeys (monkey $M P=0.042$, monkey $G P=0.13$ ). Interestingly, we found significant
Main sequence for delayed-saccades

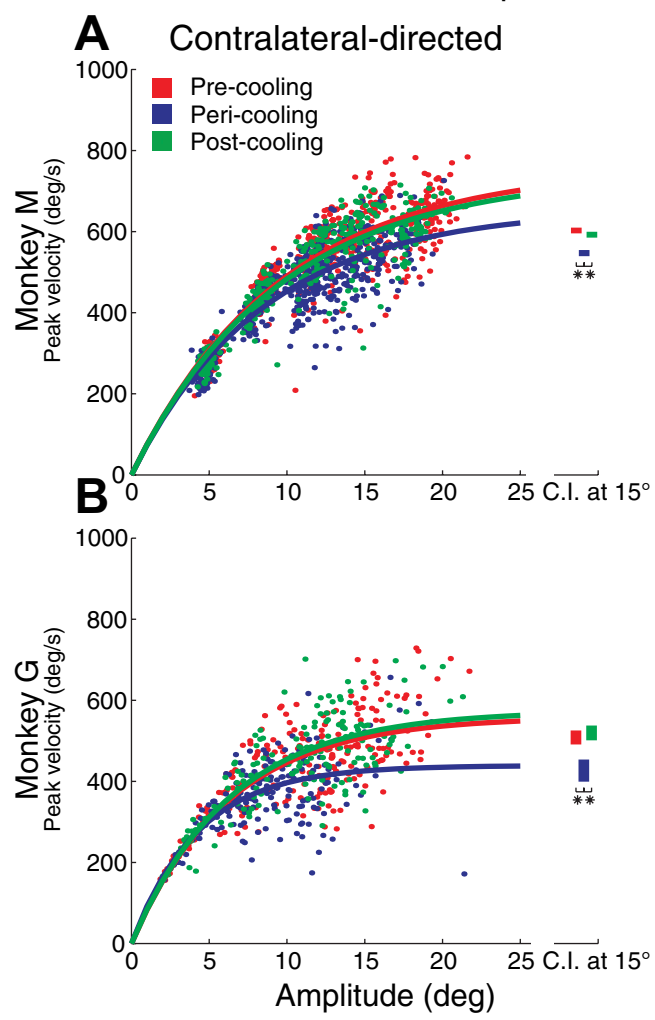

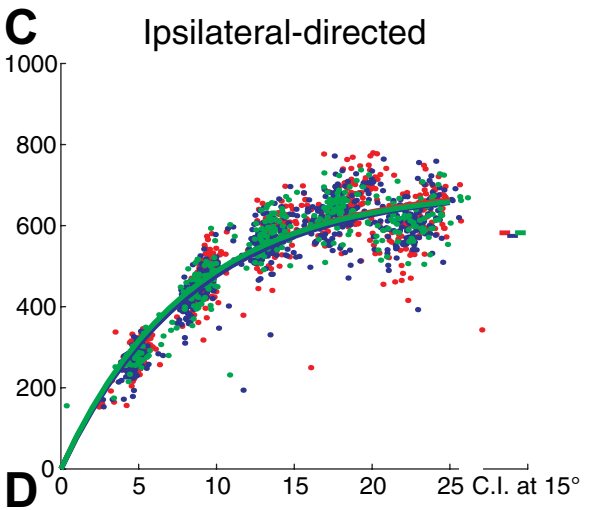

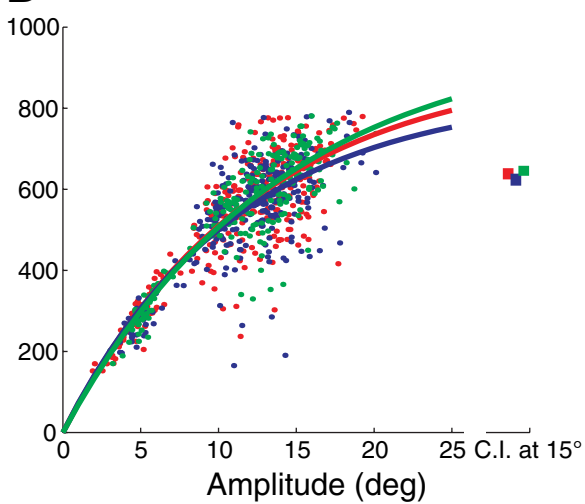

Fig. 12. Peak velocity-amplitude main sequence relationship for delayed saccade main sequence. Same format as Fig. 11. 
Fig. 13. Comparison of errors in neglect or misdirected saccades between memory and delayed saccade trials. Same format as Fig. 8.
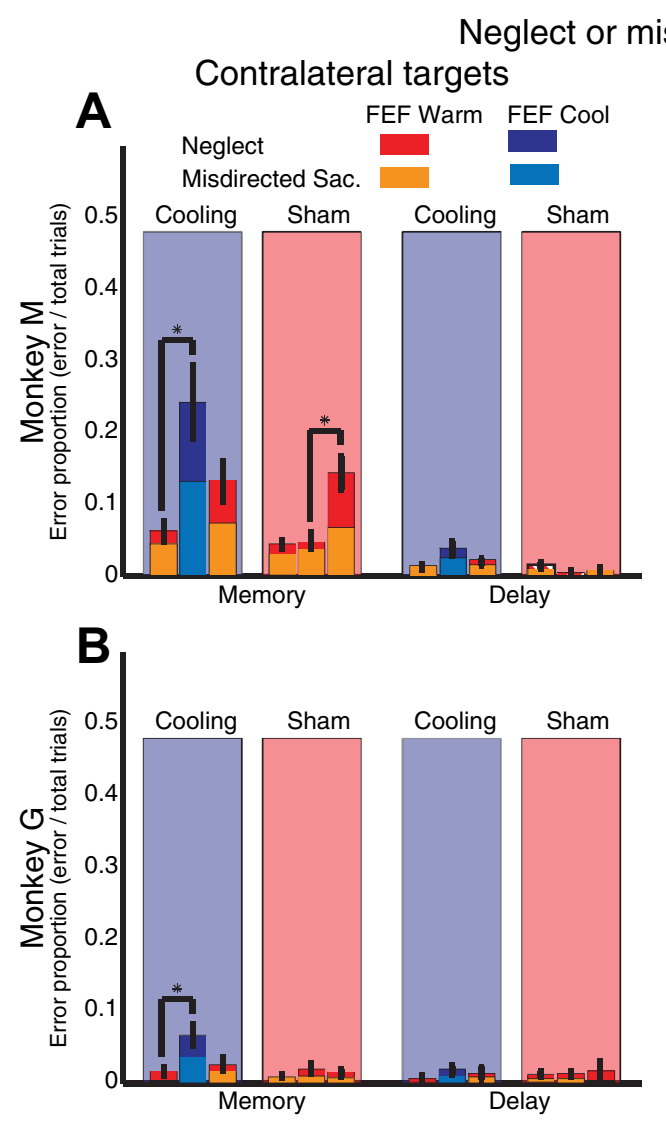
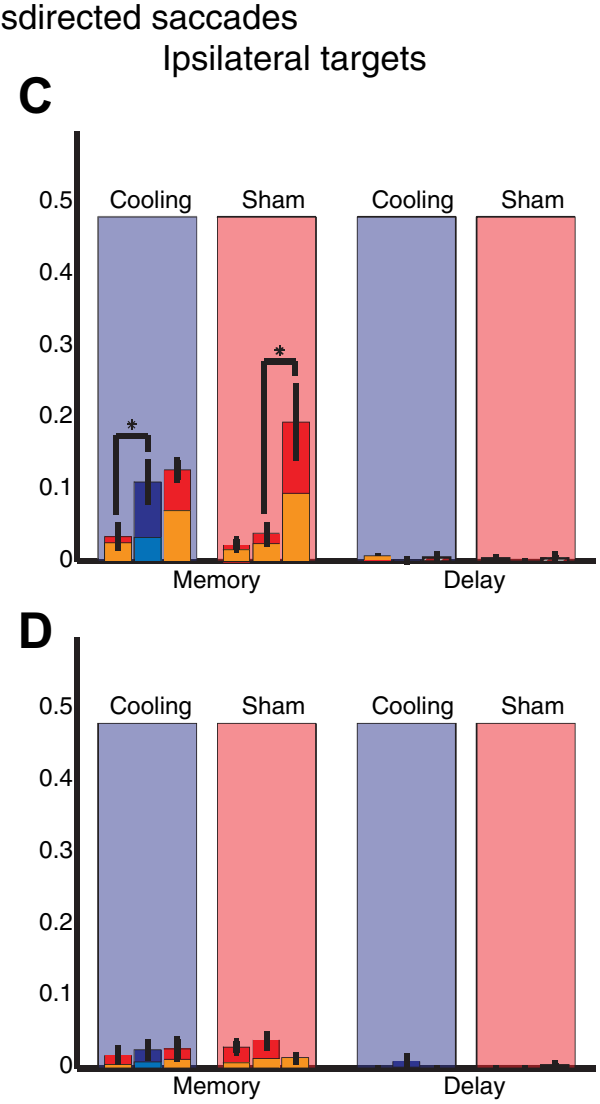

increases in errors only for monkey $M$ 's memory saccade trials toward ipsilateral cues (Fig. 13, $C$ and $D$ ). With a three-way ANOVA for monkey $M$ during $\mathrm{FEF} /$ sham inactivation, we found significant effects of cue locations (contra- and ipsilateral; $P<0.01$ ), tasks (memory and delayed saccades; $P<$ 0.0001 ), and conditions (cooling and sham; $P<0.0001$ ) on combined neglect and misdirected saccades error rate. We also found significant two-way interactions of cue direction and condition $(P<0.05)$ and task and condition $(P<0.005)$. A similar ANOVA analysis for monkey $G$ during FEF/sham inactivation revealed significant effects only of tasks $(P<$ 0.0001 ) on error rate. We also found a significant two-way interaction of cue direction and condition $(P<0.025)$. After FEF rewarming, monkey $M$ 's and monkey $G$ 's neglect and misdirected saccades errors considerably decreased, nearly reaching significance (monkey $M P=0.14$, monkey $G P=$ 0.07). In sum, we found that both monkeys exhibited an increased tendency upon FEF inactivation to make errors of neglect or misdirected saccades primarily in trials with contralateral, remembered cues.

Unilateral FEF inactivation had mild and inconsistent effects on premature saccade errors. Finally, we describe the effects of FEF inactivation on saccades generated prematurely (the interval for premature saccades spans from the time of cue presentation until $60 \mathrm{~ms}$ after disappearance of the fixation LED). During FEF inactivation, we observed only mild and inconsistent increases of premature saccade errors for both monkeys toward ipsilateral presented cues (Fig. 14, C and D) with the only evidence being the nearly significant increases occurring in delay saccade trials for monkey $M(P=0.05$; paired $t$-test, Bonferroni corrected for multiple comparisons).
In contrast, we found no significant increases in premature saccade errors for both monkeys in contralateral cue trials (Fig. $14, A$ and $B$ ) or any recovery upon rewarming. Although we found no significant increases across the cooling transitions, monkey $M$ had significantly greater premature saccade errors with FEF inactivation compared with the sham condition. Using a three-way ANOVA for monkey $M$ during FEF/sham inactivation, we found significant effects of cue locations (contra- and ipsilateral; $P<0.05$ ), tasks (memory and delayed saccades; $P<0.001$ ), and conditions (cooling and sham; $P<$ $0.001)$ on premature saccade error rate. In contrast, a similar analysis for monkey $G$ during $\mathrm{FEF} /$ sham inactivation revealed significant effects only of tasks $(P<0.0001)$ on error rate.

Summary of saccadic deficits for memory and delayed saccade task. We used the interleaved memory and delayed saccade task during unilateral FEF inactivation to compare saccadic deficits and performance errors toward remembered and persistent visual cues and to investigate the velocityamplitude main sequence relationship. Unilateral FEF inactivation increased bilateral-directed SRTs preferentially toward remembered cues, decreased peak velocities of all contraversive saccades, and increased performance errors. We also investigated saccade accuracy in this task, and found increases in both monkeys' saccade targeting error and end-point scatter during FEF inactivation primarily for contraversive saccades. An analysis of performance errors during FEF inactivation revealed that monkeys tended to neglect or look in the wrong direction of extinguished, contralateral cues and, surprisingly, had few prematurely generated saccades toward ipsilateral cues. 


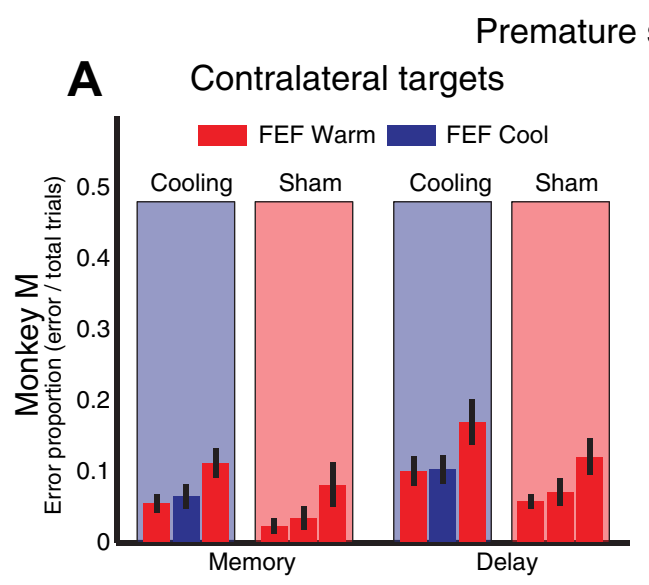

\section{Ipsilateral targets}

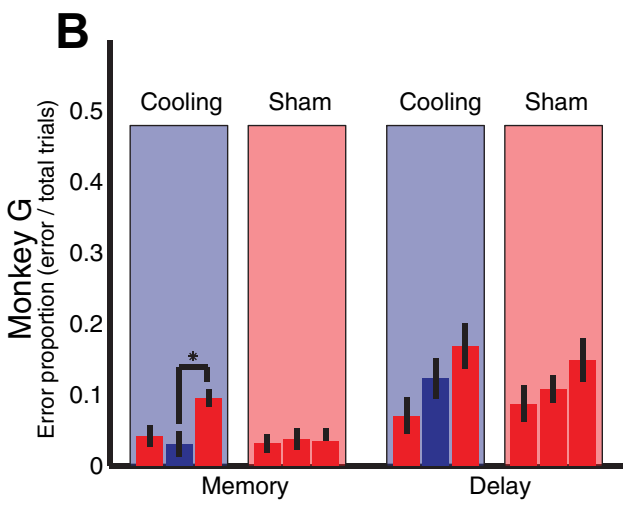

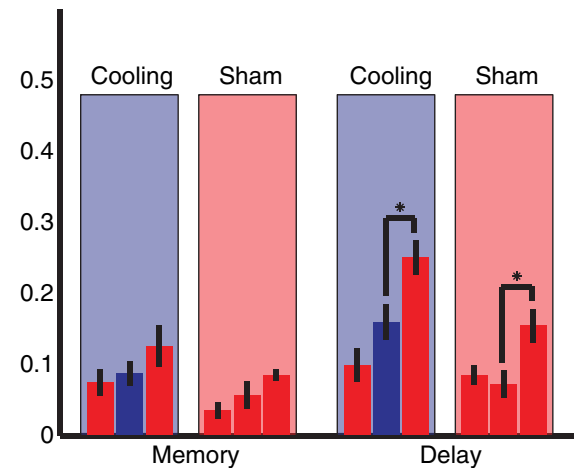

D

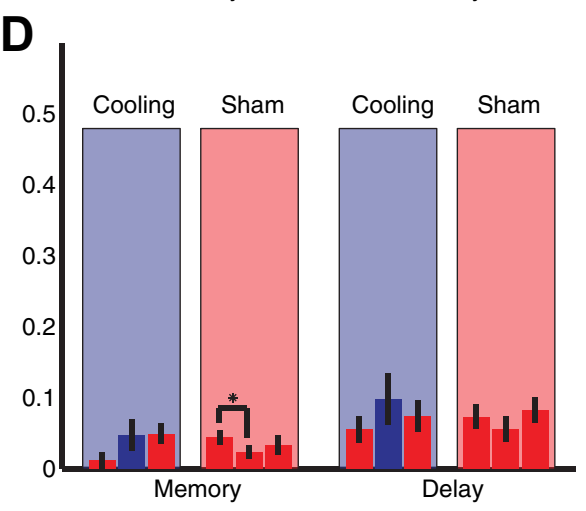

Fig. 14. Comparison of premature saccades for memory and delayed saccades. Same format as Fig. 6. Values represent the proportion of error trials divided by total trials in a session (mean $\pm \mathrm{SE}$ ratio).

\section{DISCUSSION}

We examined the effects of a large and reversible FEF inactivation on saccadic behavior separate from any long-term recovery. To accomplish this, we analyzed the saccadic deficits and performance errors in three saccade tasks before, during, and after reversible cryogenic inactivation of the unilateral FEF. We observed many of the contralateral saccadic deficits and neglect errors expected from previous reversible pharmacological inactivation studies, with greater deficits accompanying tasks with a greater working memory load. Importantly, we also observed consistent, albeit smaller, increases in RTs to ipsilateral targets that have not been previously reported. In addition, we found that premature ipsilateral saccade errors only slightly increased with FEF inactivation, in contrast to what had been expected from previous results with pharmacological inactivation. Together, these results add to the body of knowledge concerning the functional contribution of the FEF to saccades in both directions and attest to the differential effect of inactivating different volumes of the FEF.

Comparison of cryogenic inactivation to pharmacological inactivation and lesions studies. Cryogenic inactivation provides the dual advantages of inactivating a large volume of tissue in a reversible manner. On the basis of the dimensions of our constructed cryoloops and assuming that $3{ }^{\circ} \mathrm{C}$ inactivates the entire depth of the gray matter, we estimated that cooling inactivated a volume of $162 \mathrm{~mm}^{3}$. This volume is much larger than that assumed to be inactivated by pharmacological means ( $\sim 14-33 \mathrm{~mm}^{3}$ with a radius of $1-2 \mathrm{~mm}$ from the injection site; Dias and Segraves 1999; Sommer and Tehovnik 1997) and is comparable to studies using permanent ablations $\left(\sim 125 \mathrm{~mm}^{3}\right.$ circumscribing a triangular surface region of $6 \mathrm{~mm}$ along both the IA and SA of the arcuate sulcus with a depth of $8 \mathrm{~mm}$ into the arcuate sulcus; Bruce et al. 1985; Schiller and Chou 1998).

In light of the large volume that is presumably inactivated, the residual abilities that both animals displayed in generating contraversive saccades may seem somewhat surprising. Indeed, the residual abilities for animals to generate contraversive saccades following permanent ablations of the FEF are usually attributed to intact oculomotor areas taking over via parallel pathways (Schiller et al. 1980). While plastic recovery undoubtedly plays a role after permanent ablation, it is also clear that the oculomotor system can continue to operate during large but reversible inactivation of the FEF.

A methodological consideration inherent to cryogenic inactivation is that of loop placement. Our protocol involved inserting two loops oriented medial and lateral from the spur of the arcuate sulcus, which corresponds to the superior and inferior aspects of the arcuate sulcus, respectively. We selected this strategy based on previous literature and on the basis of the location of the other FEF for monkey $M$ as determined in a previous study (Elsley et al. 2007). One caveat in this strategy is the unintended inactivation of adjacent areas outside of the traditional FEF. Our loops were specifically designed to inactivate tissue within the arcuate sulcus, and hence inactivation did not extend to the premotor oculomotor regions described by Fujii and colleagues $(1998,2000)$ that lie on the gyri either posterior to the IA of the arcuate (Fujii et al. 1998) or medial to the SA of the arcuate sulcus (Fujii et al. 2000), between the frontal and supplementary eye fields. Cooling within the arcuate sulcus also did not extend to the premotor regions that lie on the gyrus between the arcuate and central sulci, from where a variety of defensive or multisegmental movements that can 
include an oculomotor component can be evoked via microstimulation (Boulanger et al. 2009; Graziano et al. 2002). Cooling the loops certainly inactivated the posterior bank of the arcuate sulcus, and while we cannot completely rule out the possibility that some of our observed deficits may be related to this, previous studies suggest that this area is not critical for saccade generation. Monkeys with either permanent (Rizzolatti et al. 1983) or reversible (Schieber 2000) lesions to premotor areas found within the posterior bank of the arcuate sulcus do not display the constellation of oculomotor deficits that we observed here, even though such lesions perturbed skeletomotor behaviors in a variety of tasks. It is possible that inactivated tissue extended into the fundus, which has been linked to smooth pursuit eye movements (Gottlieb et al. 1994) and from where $\sim 20 \%$ of neurons have presaccade responses (Tanaka and Fukushima 1998). However, muscimol inactivation of FEF sites physiologically characterized to be related to smooth pursuit severely compromised smooth pursuit without influencing saccades (Shi et al. 1998). Thus, although functional imaging and neuroanatomical techniques demonstrate that a large portion of the premotor cortex is active during visually guided saccades (Koyama et al. 2004; Moschovakis et al. 2004), the preponderance of evidence from other studies leads us to think that the majority of the saccadic deficits we observed arise from cryogenic inactivation of the anterior bank of the arcuate sulcus.

Changes to contraversive saccade behavior: spatial specificity and impact on saccade RT, accuracy, and saccade velocity. In both tasks, unilateral FEF inactivation consistently decreased saccade accuracy (manifested as increased hypometria and/or end-point scatter), decreased saccade velocities, and increased SRTs. This triad of contralateral saccadic deficits is commonly seen after either pharmacological FEF inactivation (Dias et al. 1995; Dias and Segraves 1999; Sommer and Tehovnik 1997) or ablations in monkeys (Collin et al. 1982; Deng et al. 1986; Latto and Cowey 1971; Lynch 1992; Schiller et al. 1980; Schiller and Chou 1998; van der Steen et al. 1986) and also in human patients presenting with unilateral loss of the FEF (Gaymard et al. 1999; Pierrot-Deseilligny et al. 1991; Rivaud et al. 1994).

The spatial distribution of saccadic deficits we observed encompassed contraversive saccades with or without an oblique component, with smaller deficits accompanying smaller saccade amplitudes. Such relative sparing of smaller-amplitude saccades may relate to the logarithmic coding of oculocentric space, with proportionally more tissue devoted to smaller-amplitude saccades (Bruce and Goldberg 1985; Schwartz 1980). Alternatively (or perhaps additionally), our protocol for cryoloop placement may not have been optimal to influence the more ventrolateral portions of the FEF preferentially involved in small-amplitude saccades (Bruce and Goldberg 1985).

In all tasks, we consistently found that FEF inactivation decreased contraversive saccade peak velocities, with greater decreases occurring if the target was extinguished during the memory versus delayed saccade paradigm. Previous studies using pharmacological inactivation have observed such contraversive decreases in peak velocity (Dias and Segraves 1999), although others had only inconsistent effects (Sommer and Tehovnik 1997). Dias and Segraves (1999) postulated that the lack of effects on saccade dynamics found by Sommer and
Tehovnik (1997) was due to generally slower peak velocities, possibly from the dark environment, which could result in less peak velocity difference between before and after FEF inactivation. This discrepancy may also be due to the use of primarily lidocaine in the Sommer and Tehovnik (1997) study versus muscimol in the Dias and Segraves (1999) study. Regardless of the mechanism, our results confirm that a large volume of FEF inactivation influences saccade dynamics.

All contralateral saccadic deficits tended to be greater in the memory-guided versus delayed saccade paradigm, attesting to the FEF's increased role in more cognitively demanding tasks. This result is also consistent with the greater and longer-lasting performance deficits for memory-guided saccades compared with visually guided saccades after either ablations (Deng et. al. 1986) or pharmacological inactivation (Dias and Segraves 1999; Sommer and Tehovnik 1997) of FEF. Cognitive signals in the FEF, including delay-period activity, appear to play a prominent role in the sensorimotor transformation for saccades. Indeed, a sample of FEF neurons projecting to the superior colliculus (SC) contain visuomotor delay activity that is modulated by Go/Nogo task instructions for only memoryguided saccades; therefore this delay activity may be a correlate of working memory (Sommer and Wurtz 2001).

Finally, a surprising aspect of our results was the negligible effect of unilateral cryogenic inactivation on gross fixation behavior, particularly given the previous pharmacological inactivation study by Dias and Segraves (1999), which showed increased scatter during fixation and modest ipsilateral shifts in spontaneous eye position, neither of which was apparent in either monkey. In addition, Sommer and Tehovnik (1997) reported that monkeys with pharmacological FEF inactivations had difficulty maintaining fixation of peripheral contralateral targets. Fixation-related neurons and sites where electrical stimulation increased bilateral-directed saccade RTs tend to be preferentially located more lateral in the arcuate sulcus and ventrally toward the fundus (Burman and Bruce 1997; Izawa et al. 2004, 2009). This suggests that either our volume of inactivation did not encompass these lateral and/or ventral FEF subregions or a more focal FEF inactivation preferentially causes fixation-related deficits. Note, however, that we did not require our monkeys to fixate eccentrically for a sustained period of time, as was done by Sommer and Tehovnik (1997); hence it is possible that fixation deficits could have been revealed in our monkeys had we used a modified task or required different behaviors.

Increased ipsiversive SRTs without concomitant changes to accuracy or saccade velocity. In addition to contralateral saccadic deficits, large and reversible inactivation of the unilateral FEF also impacted the generation of ipsiversive saccades. Importantly, the impact of inactivation is restricted to increased RTs, with negligible impact on saccade accuracy or dynamics. In all tasks for both monkeys we observed significant increases in ipsiversive SRTs of $\sim 25-50 \mathrm{~ms}$, and while this is less than the RT increase for contraversive saccades, it always exceeded the RT increases observed in the same time interval for sham cooling sessions. Previous FEF inactivation studies have reported only subtle or negligible effects on ipsiversive saccades with either lesions (Collin et al. 1982; Deng et. al. 1986; Latto and Cowey 1971; Lynch 1992; Schiller et al. 1980; Schiller and Chou 1998; van der Steen et al. 1986) or injected pharmaceuticals (Dias and Segraves 1999; Sommer 
and Tehovnik 1997), leading us to speculate that the ipsilateral saccade deficits are unique to large and reversible FEF inactivation.

Two potential mechanisms could produce increased ipsilateral SRTs upon inactivation without changing metrics or dynamics. One mechanism could be the relatively sparse distribution of FEF neurons that exhibit ipsilateral responses fields (Bruce and Goldberg 1985). Because of such a sparse distribution, the functional contribution of these neurons to ipsiversive saccades would only be revealed with a large volume of inactivation, which may be why pharmacological inactivation studies have not observed consistent effects. The functional contribution of ipsilateral-related FEF neurons may be also fundamentally different from the canonical contralateral saccade-related neurons in the FEF; specifically, ipsilateral-related FEF neurons may exert their influence on ipsiversive SRTs through projections that bypass the SC (e.g., indirectly via basal ganglia or to the brain stem oculomotor areas; see Predictions of neuronal activity in downstream oculomotor areas below), influencing target selection or saccade initiation without influencing the vigor or representation of the oculomotor drive in the brain stem. Although recent work by Crapse and Sommer (2009) has identified some of the inputs to these ipsilateral-related FEF neurons, their functional contribution to oculomotor control remains to be determined.

Alternatively, a large lesion of one FEF may produce widespread disinhibition of the other FEF, with the consequence of somewhat paradoxically prolonging the target selection process for ipsilateral saccades. The FEFs in each hemisphere have connections with each other (Pandya and Vignolo 1971), and the influence of these connections is thought to be mostly inhibitory (Schlag et al. 1998; Seidemann et al. 2002). Our observation of broadly increased ipsiversive SRTs is all the more surprising in light of what is usually thought about interhemispheric FEF communication, which would have predicted decreased ipsiversive SRTs. However, the FEF is known to be a key area for saccade target selection (for review, see Schall 2002), instantiating a gradual discrimination between representations of a target from distractors. Such selection is thought to involve inter- and intrahemispheric FEF networks, supporting cooperative or competitive interactions between FEF neurons that share overlapping or nonoverlapping response fields (Cohen et al. 2010). If a large portion of one FEF is broadly disinhibited because of a large-volume inactivation of the other FEF it would presumably take longer for the target selection processes to resolve into a single choice, but once resolved the saccade would have normal metrics and dynamics. In contrast, if a focal portion of one FEF is inactivated, only the mirror location of the other FEF would be disinhibited, leading to shorter ipsilateral RTs only for that location.

These two explanations need not be mutually exclusive. What they do provide is a potential explanation of why increased ipsilateral SRTs are unique to large and reversible FEF lesions and occur without concomitant changes in saccade metrics or dynamics.

Increased contralateral neglect but no increased tendency for premature ipsiversive saccades. The functional contribution of the FEF to saccades can also be revealed through an analysis of various error types in different tasks. We observed a markedly increased tendency for both monkeys to either neglect (i.e., not respond) or look in the opposite direction to contralateral-presented stimuli in the memory-guided saccade task. This tendency was greatly reduced in both monkeys if the stimulus remained on in the delayed-saccade task, reinforcing the increased contribution of the FEF in tasks with a greater working memory requirement. Previous FEF studies have made similar observations with pharmacological inactivation (Dias et al. 1995; Dias and Segraves 1999; Sommer and Tehovnik 1997) or ablations (Collin et al. 1982; Deng et. al. 1986; Latto and Cowey 1971; Lynch 1992; Schiller et al. 1980; Schiller and Chou 1998; van der Steen et al. 1986). These observations of neglect do not appear attributable to inabilities in detecting or remembering contralateral cues. Using monkeys trained in a memory-guided saccade task that spatially dissociated the saccadic response from cue location, Lee and colleagues (2012) found that pharmacological FEF inactivation resulted in no marked differences in monkeys' performance for spatially dissociated responses following contralateral cue presentation. Alternatively, when the required responses were not spatially dissociated from contralateral cues, monkeys frequently neglected to generate saccades or looked in the wrong direction, which is in agreement with our own results.

In contrast to previous pharmacological FEF inactivation studies, we found essentially no consistent tendency for either monkey to generate premature ipsiversive saccades during FEF inactivation in the memory-guided or delayed saccade paradigms, particularly compared with the results with sham inactivation (Fig. 14). To put our observations in perspective with previous results, Sommer and Tehovnik (1997) reported that the tendency to generate premature saccades to ipsilateral cues increased from $\sim 5 \%$ before pharmacological inactivation to $\sim 50 \%$ during FEF inactivation in a memory-guided saccade task. Similarly, Dias and Segraves (1999) reported that the tendency to generate premature saccades to ipsilateral stimuli in a similar task increased progressively from $<2 \%$ before inactivation to almost $100 \% \sim 2 \mathrm{~h}$ after muscimol injection. The marked differences between our results and those of studies using pharmacological inactivation appear to be another example of the effect of FEF inactivation volume; a more focal FEF inactivation results in increased difficulty in suppressing inappropriate saccades toward ipsilateral cues at the mirror location.

Predictions of neuronal activity in downstream oculomotor areas. The triad of contralateral saccadic deficits after largevolume unilateral FEF inactivation is largely consistent with the robust and topographically organized projections from FEF to the SC (Leichnetz et al. 1981; Sommer and Wurtz 2000). FEF neurons do not appear to encode saccadic metrics and dynamics per se (Segraves and Park 1993), although there are correlations with SRT (Hanes et al. 1998; Heitz and Schall 2012; Segraves and Park 1993). Instead, oculocentric signals relayed to downstream oculomotor areas convert these signals into saccadic vectors (Dassonville et al. 1992). Neural activity within the intermediate and deep layers of the SC relates to saccade timing, metrics, and dynamics (Mays and Sparks 1980; Munoz et al. 2000), and reversible inactivation or ablations of the SC produce the same triad of saccadic deficits (Aizawa and Wurtz 1998; Albano et al. 1982; Albano and Wurtz 1982; Cavanaugh et al. 2012b; Hikosaka and Wurtz 1983, 1986; Quaia and Wurtz 1998; Schiller et al. 1980; Wurtz and Goldberg 1972). Given the contralateral saccadic deficits we observed, it is likely that saccade-related activity in the ipsilateral 
SC takes longer to reach threshold, is spatially displaced and variable from the representation of the target before cooling, and reaches a lower peak firing rate. What remains an open question is how $\mathrm{SC}$ activity outside of the perisaccadic interval is influenced by FEF inactivation, particularly during memoryguided and delayed saccades; our preliminary results indicate that visual and delay-period activity in the ipsilateral SC largely decreases during FEF inactivation (Peel et al. 2012), which suggests that the FEF functionally contributes to all aspects of ipsilateral SC activity.

In addition to the robust and topographic projections to the $\mathrm{SC}$, descending projections from the FEF also go through the basal ganglia (Kunzle and Akert 1977) and other brain stem centers downstream from the SC. The direct influence of the basal ganglia on brain stem saccadic activity is thought to be predominantly relayed through the substantia nigra pars reticulata $(\mathrm{SNr})$, which has projections to the ipsilateral and contralateral SC via uncrossed (Chevalier et al. 1981; Graybiel and Ragsdale 1978; Hikosaka and Wurtz 1983; Jayaraman et al. 1977) and crossed (Beckstead 1981; Jiang et al. 2003; Liu and Basso 2008) pathways, respectively. Although both the uncrossed and crossed projections are inhibitory, Jiang and colleagues (2003) found that these projections also differ in several respects (e.g., spatial distributions of $\mathrm{SNr}$ neurons, spontaneous activity, conduction velocities, and response fields); therefore, they suggested that coordination of these pathways (i.e., inhibition by crossed pathway and disinhibition by uncrossed pathway) could facilitate presaccadic activity in the SC. Previously, Hikosaka and Wurtz (1983) had investigated the influence of the $\mathrm{SNr}$ on presaccadic activity in the ipsilateral SC (i.e., uncrossed pathway). Liu and Basso (2008) showed that electrical stimulation in $\mathrm{SNr}$ transiently decreases presaccadic activity in both contralateral and ipsilateral SC neurons. While the functional role of the crossed pathway for oculomotor behavior remains to be determined, the crossed pathway provides a substrate by which FEF activity can indirectly influence the SC on the other side. In light of our results, we speculate that any influence on the contralateral SC would be limited to the timing of the saccaderelated activity.

A similar degree of uncertainty exists when trying to predict the impact of FEF inactivation on the signals conveyed directly to the brain stem, downstream from the SC. FEF neurons project to the ipsilateral oculomotor regions of the pons, including both the omnipause and saccadic burst generation regions (Huerta et al. 1986; Leichnetz et al. 1984; Schnyder et al. 1985; Segraves 1992; Stanton et al. 1988a, 1988b), and contain functional signals that largely resemble those sent directly to the SC (Segraves 1992; Segraves and Goldberg 1987). However, the ability to electrically evoke saccades directly from the FEF depends on the integrity of the SC (Hanes and Wurtz 2001), suggesting either that the direct projections from the FEF to the oculomotor brain stem are insufficient to evoke saccades or that an additional signal from the $\mathrm{SC}$ is required in downstream structures. Alternatively, FEF signals that are sent directly to the brain stem may participate in ongoing saccadic preparation via cortico-cerebellar loops. The first part of the cortico-cerebellar loop consists of a corticopontocerebellar disynaptic pathway that innervates cerebellar hemispheric lobule VII (Huerta et al. 1986; Schmahmann and Pandya 1997; Xiong et al. 2002) and the adjacent dentate nucleus via Purkinje neurons (Xiong et al. 2002). The loop is closed by ascending disynaptic projections from the dentate nucleus to the FEF through the ventrolateral nucleus of the thalamus (Lynch et al. 1994), although the dentate nucleus also projects to several other oculomotor areas, including the SC (May et al. 1990) and LIP (Prevosto et al. 2010). Using reversible inactivation of ventrolateral nucleus, Tanaka (2006) reported increased RTs of self-timed saccades and suggested that these ascending projections back to cortex are important for the timing of self-triggered saccades. Subsequently, Tanaka (2007) found delay-period activity in neurons of the ventrolateral nucleus, and this activity was correlated to saccade generation when tasks required internal monitoring of time (i.e., self-triggered saccades) or were associated with predictive cues related to saccade timing (i.e., disappearance of fixation light in delayed or memory saccades). Recently, Ashmore and Sommer (2013) suggested that one probable source of this delay-period activity is the dentate nucleus. They found delay-period activity in neurons of the dentate nucleus, and this activity was related to the initiation and directionality (but not accuracy or dynamics) of self-triggered saccades. One implication of this finding is that cortico-cerebellar loops appear to play a role in self-triggered saccades; therefore FEF inactivation may result in impairments of self-triggered saccades by removing a key source of inputs and recipient area of signals from corticocerebellar loops.

How then do we explain the changes to ipsiversive saccade behavior? The increase in ipsiversive SRTs without concomitant changes in saccade metrics or dynamics, as well as the lack of any consistent increase in the tendency to generate premature saccades to ipsilateral stimuli in either the memory-guided or delayed saccade task, suggests a mechanism whose influence is restricted to saccade timing rather than saccade generation per se. Such changes may speak to the functional contribution of ipsilateral-related neurons in the FEF contributing to saccade timing but not metrics and dynamics. Although speculative, altered signaling from these neurons either through the basal ganglia (e.g., delayed disinhibition via the crossed pathway) or directly to the oculomotor brain stem (e.g., a delayed pause of the omnipause neurons or impairments to corticocerebellar loops) could explain our results. Alternatively, as described above, large-volume FEF inactivation may cause broad disinhibition of the contralateral FEF neurons, disrupting the balance of cooperative and competitive interactions among local FEF neurons in the target selection process.

Differential effects of focal versus large-volume inactivation? One implication of our results compared with those obtained with more focal, pharmacologically mediated inactivation is that the volume of inactivation may have an important impact on both contralateral and ipsiversive saccades. In particular, focal inactivation may promote a degree of disinhibition in the mirroring location via a loss of interhemispheric inhibition that is not obtained with large-volume inactivation. Large-volume inactivation may exert a different impact on saccade behavior, perhaps because of its proportionally greater impact on functional classes of neuron (like ipsilateral-related neurons) that have a more dispersed distribution in the FEF, because of differences in interhemispheric inhibition, or because of the properties of downstream circuits (e.g., in the basal ganglia). To further complicate matters, finer details of receptive field structures in the FEF continue to emerge (e.g., Cavanaugh et al. 2012a), making it even harder to predict the comparative 
changes in the FEF with progressively greater volumes of inactivation. An appreciation of the potential differences between focal and large-volume inactivation is particularly relevant for experiments that aim to record in one structure while inactivating the other; in the oculomotor network in particular, the problem with aligning the recorded and inactivated response fields is largely avoided with large-volume inactivation. We are currently conducting such studies to directly investigate the impact of large-volume FEF inactivation on neuronal activity in the SC.

\section{ACKNOWLEDGMENTS}

The authors thank Katherine Green for animal monitoring during the surgical procedures; Kevin Barker for engineering assistance; Pam Nixon and Amee Hall for assistance in constructing and placement of cryoloops; and Suryadeep Dash and Chao Gu for comments on an earlier version of the manuscript.

\section{GRANTS}

T. R. Peel was supported by an Ontario Graduate Scholarship and funding from the Natural Sciences and Engineering Research Council of Canada CREATE grant. This work was supported by an operating grant from the Canadian Institutes of Health Research (CIHR) to B. D. Corneil (MOP 93796).

\section{DISCLOSURES}

No conflicts of interest, financial or otherwise, are declared by the author(s).

\section{AUTHOR CONTRIBUTIONS}

Author contributions: T.R.P., S.G.L., and B.D.C. conception and design of research; T.R.P. performed experiments; T.R.P. analyzed data; T.R.P., K.J., S.G.L., and B.D.C. interpreted results of experiments; T.R.P. prepared figures; T.R.P. and B.D.C. drafted manuscript; T.R.P., K.J., S.G.L., and B.D.C. edited and revised manuscript; T.R.P., K.J., S.G.L., and B.D.C. approved final version of manuscript.

\section{REFERENCES}

Aizawa H, Wurtz RH. Reversible inactivation of monkey superior colliculus. I. Curvature of saccadic trajectory. J Neurophysiol 79: 2082-2096, 1998.

Albano JE, Mishkin M, Westbrook LE, Wurtz RH. Visuomotor deficits following ablation of monkey superior colliculus. J Neurophysiol 48: 338 351, 1982.

Albano JE, Wurtz RH. Deficits in eye position following ablation of monkey superior colliculus, pretectum, and posterior-medial thalamus. $J$ Neurophysiol 48: 318-337, 1982.

Ashmore RC, Sommer MA. Delay activity of saccade-related neurons in the caudal dentate nucleus of the macaque cerebellum. J Neurophysiol 109: 2129-2144, 2013.

Beckstead RM, Edwards SB, Frankfurter A. A comparison of the intranigral distribution of nigrotectal neurons labeled with horseradish peroxidase in the monkey, cat, and rat. J Neurosci 1: 121-125, 1981

Boulanger M, Bergeron A, Guitton D. Ipsilateral head and centring eye movements evoked from monkey premotor cortex. Neuroreport 20: 669673, 2009.

Bruce CJ, Goldberg ME. Primate frontal eye fields. I. Single neurons discharging before saccades. J Neurophysiol 53: 603-635, 1985.

Bruce CJ, Goldberg ME, Bushnell MC, Stanton GB. Primate frontal eye fields. II. Physiological and anatomical correlates of electrically evoked eye movements. J Neurophysiol 54: 714-734, 1985.

Burman DD, Bruce CJ. Suppression of task-related saccades by electrical stimulation in the primate's frontal eye field. J Neurophysiol 77: 2252-2267, 1997.

Cavanaugh J, Joiner WM, Wurtz RH. Suppressive surrounds of receptive fields in monkey frontal eye field. J Neurosci 32: 12284-12293, 2012a.
Cavanaugh J, Monosov IE, McAlonan K, Berman R, Smith MK, Cao V, Wang KH, Boyden ES, Wurtz RH. Optogenetic inactivation modifies monkey visuomotor behavior. Neuron 76: 901-907, 2012 b.

Chevalier G, Deniau JM, Thierry AM, Feger J. The nigro-tectal pathway. An electrophysiological reinvestigation in the rat. Brain Res 213: 253-263, 1981.

Cohen JY, Crowder EA, Heitz RP, Subraveti CR, Thompson KG, Woodman GF, Schall JD. Cooperation and competition among frontal eye field neurons during visual target selection. J Neurosci 30: 3227-3238, 2010.

Collin NG, Cowey A, Latto R, Marzi C. The role of frontal eye-fields and superior colliculi in visual search and non-visual search in rhesus monkeys. Behav Brain Res 4: 177-193, 1982.

Crapse TB, Sommer MA. Frontal eye field neurons with spatial representations predicted by their subcortical input. J Neurosci 29: 5308-5318, 2009.

Dassonville P, Schlag J, Schlag-Rey M. The frontal eye field provides the goal of saccadic eye movement. Exp Brain Res 89: 300-310, 1992.

Deng SY, Goldberg ME, Segraves MA, Ungerleider LG, Mishkin M. The effect of unilateral ablation of the frontal eye fields on saccadic performance in the monkey. In: Adaptive Processes in the Visual and Oculomotor Systems, edited by Keller EL, Zee DS. Oxford, UK: Pergamon, 1986, p. 201-208.

Dias EC, Bruce CJ. Physiological correlate of fixation disengagement in the primate's frontal eye field. J Neurophysiol 72: 2532-2537, 1994.

Dias EC, Kiesau M, Segraves MA. Acute activation and inactivation of macaque frontal eye field with GABA-related drugs. J Neurophysiol 74: 2744-2748, 1995.

Dias EC, Segraves MA. Muscimol-induced inactivation of monkey frontal eye field: effects on visually and memory-guided saccades. $J$ Neurophysiol 81: 2191-2214, 1999.

Elsley JK, Nagy B, Cushing SL, Corneil BD. Widespread presaccadic recruitment of neck muscles by stimulation of the primate frontal eye fields. J Neurophysiol 98: 1333-1354, 2007.

Fujii N, Mushiake H, Tanji J. An oculomotor representation area within the ventral premotor cortex. Proc Natl Acad Sci USA 95: 12034-12037, 1998.

Fujii N, Mushiake H, Tanji J. Rostrocaudal distinction of the dorsal premotor area based on oculomotor involvement. J Neurophysiol 83: 1764-1769, 2000.

Funahashi S, Bruce CJ, Goldman-Rakic PS. Neuronal activity related to saccadic eye movements in the monkey's dorsolateral prefrontal cortex. $J$ Neurophysiol 65: 1464-1483, 1991.

Gaymard B, Ploner CJ, Rivaud-Pechoux S, Pierrot-Deseilligny C. The frontal eye field is involved in spatial short-term memory but not in reflexive saccade inhibition. Exp Brain Res 129: 288-301, 1999.

Gottlieb JP, MacAvoy MG, Bruce CJ. Neural responses related to smoothpursuit eye movements and their correspondence with electrically elicited smooth eye movements in the primate frontal eye field. $J$ Neurophysiol 72 : 1634-1653, 1994.

Graybiel AM, Ragsdale CW Jr. Histochemically distinct compartments in the striatum of human, monkeys, and cat demonstrated by acetylthiocholinesterase staining. Proc Natl Acad Sci USA 75: 5723-5726, 1978.

Graziano MS, Taylor CS, Moore T. Complex movements evoked by microstimulation of precentral cortex. Neuron 34: 841-851, 2002.

Halsband U, Passingham R. The role of premotor and parietal cortex in the direction of action. Brain Res 240: 368-372, 1982.

Hanes DP, Patterson WF 2nd, Schall JD. Role of frontal eye fields in countermanding saccades: visual, movement, and fixation activity. $J \mathrm{Neu}$ rophysiol 79: 817-834, 1998.

Hanes DP, Wurtz RH. Interaction of the frontal eye field and superior colliculus for saccade generation. J Neurophysiol 85: 804-815, 2001.

Heitz RP, Schall JD. Neural mechanisms of speed-accuracy tradeoff. Neuron 76: 616-628, 2012.

Hikosaka O, Wurtz RH. Effects on eye movements of a GABA agonist and antagonist injected into monkey superior colliculus. Brain Res 272: $368-$ 372, 1983.

Hikosaka O, Wurtz RH. Saccadic eye movements following injection of lidocaine into the superior colliculus. Exp Brain Res 61: 531-539, 1986.

Huerta MF, Krubitzer LA, Kaas JH. Frontal eye field as defined by intracortical microstimulation in squirrel monkeys, owl monkeys, and macaque monkeys. I. Subcortical connections. J Comp Neurol 253: 415-439, 1986. 
Izawa Y, Suzuki H, Shinoda Y. Suppression of visually and memory-guided saccades induced by electrical stimulation of the monkey frontal eye field. II. Suppression of bilateral saccades. J Neurophysiol 92: 2261-2273, 2004.

Izawa Y, Suzuki H, Shinoda Y. Response properties of fixation neurons and their location in the frontal eye field in the monkey. J Neurophysiol 102: 2410-2422, 2009.

Jayaraman A, Batton RR 3rd, Carpenter MB. Nigrotectal projections in the monkey: an autoradiographic study. Brain Res 135: 147-152, 1977.

Jiang H, Stein BE, McHaffie JG. Opposing basal ganglia processes shape midbrain visuomotor activity bilaterally. Nature 423: 982-986, 2003.

Johnston K, Koval MJ, Lomber SG, Everling S. Macaque dorsolateral prefrontal cortex does not suppress saccade-related activity in the superior colliculus. Cereb Cortex (January 10, 2013). doi:10.1093/cercor/bhs424.

Koyama M, Hasegawa I, Osada T, Adachi Y, Nakahara K, Miyashita Y. Functional magnetic resonance imaging of macaque monkeys performing visually guided saccade tasks: comparison of cortical eye fields with humans. Neuron 41: 795-807, 2004.

Kunzle H, Akert K. Efferent connections of cortical, area 8 (frontal eye field) in Macaca fascicularis. A reinvestigation using the autoradiographic technique. J Comp Neurol 173: 147-164, 1977.

Latto R, Cowey A. Visual field defects after frontal eye-field lesions in monkeys. Brain Res 30: 1-24, 1971.

Lee KM, Ahn KH, Keller EL. Saccade generation by the frontal eye fields in rhesus monkeys is separable from visual detection and bottom-up attention shift. PLoS One 7: e39886, 2012

Leichnetz GR, Spencer RF, Hardy SG, Astruc J. The prefrontal corticotectal projection in the monkey; an anterograde and retrograde horseradish peroxidase study. Neuroscience 6: 1023-1041, 1981.

Leichnetz GR, Spencer RF, Smith DJ. Cortical projections to nuclei adjacent to the oculomotor complex in the medial dien-mesencephalic tegmentum in the monkey. J Comp Neurol 228: 359-387, 1984.

Liu P, Basso MA. Substantia nigra stimulation influences monkey superior colliculus neuronal activity bilaterally. J Neurophysiol 100: 1098-1112, 2008.

Lomber SG. The advantages and limitations of permanent or reversible deactivation techniques in the assessment of neural function. $J$ Neurosci Methods 86: 109-117, 1999.

Lomber SG, Payne BR, Horel JA. The cryoloop: an adaptable reversible cooling deactivation method for behavioral or electrophysiological assessment of neural function. J Neurosci Methods 86: 179-194, 1999.

Lynch JC. Saccade initiation and latency deficits after combined lesions of the frontal and posterior eye fields in monkeys. J Neurophysiol 68: 1913-1916, 1992.

Lynch JC, Hoover JE, Strick PL. Input to the primate frontal eye field from the substantia nigra, superior colliculus, and dentate nucleus demonstrated by transneuronal transport. Exp Brain Res 100: 181-186, 1994.

May PJ, Hartwich-Young R, Nelson J, Sparks DL, Porter JD. Cerebellotectal pathways in the macaque: implications for collicular generation of saccades. Neuroscience 36: 305-324, 1990.

Mays LE, Sparks DL. Dissociation of visual and saccade-related responses in superior colliculus neurons. J Neurophysiol 43: 207-232, 1980.

Moll L, Kuypers HG. Premotor cortical ablations in monkeys: contralateral changes in visually guided reaching behavior. Science 198: 317-319, 1977.

Moschovakis AK, Gregoriou GG, Ugolini G, Doldan M, Graf W, Guldin W, Hadjidimitrakis K, Savaki HE. Oculomotor areas of the primate frontal lobes: a transneuronal transfer of rabies virus and $\left[{ }^{14} \mathrm{C}\right]$-2-deoxyglucose functional imaging study. J Neurosci 24: 5726-5740, 2004.

Munoz DP, Dorris MC, Pare M, Everling S. On your mark, get set: brainstem circuitry underlying saccadic initiation. Can J Physiol Pharmacol 78: 934-944, 2000.

Nassi JJ, Lomber SG, Born RT. Corticocortical feedback contributes to surround suppression in V1 of the alert primate. J Neurosci 33: 8504-8517, 2013

Olfert E, Cross B, McWilliam A. Guide to the Care and Use of Experimental Animals. Ottawa, ON, Canada: Canadian Council on Animal Care, 1993.

Pandya DN, Vignolo LA. Intra- and interhemispheric projections of the precentral, premotor and arcuate areas in the rhesus monkey. Brain Res 26: 217-233, 1971.

Peel TR, Lomber SG, Corneil BD. Behavioural effects of unilateral cryogenic inactivation of primate frontal eye fields (Abstract). Soc Neurosci Abstr 2010: 779.17, 2010.

Peel TR, Lomber SG, Corneil BD. Functional contribution of the frontal eye fields to activity in the intermediate superior colliculus (Abstract). Soc Neurosci Abstr 2012: 373.10, 2012.
Pierrot-Deseilligny C, Rivaud S, Gaymard B, Agid Y. Cortical control of reflexive visually-guided saccades. Brain 114: 1473-1485, 1991.

Ponce CR, Hunter JN, Pack CC, Lomber SG, Born RT. Contributions of indirect pathways to visual response properties in macaque middle temporal area MT. J Neurosci 31: 3894-3903, 2011.

Prevosto V, Graf W, Ugolini G. Cerebellar inputs to intraparietal cortex areas LIP and MIP: functional frameworks for adaptive control of eye movements, reaching, and arm/eye/head movement coordination. Cereb Cortex 20: 214-228, 2010.

Quaia C, Aizawa H, Optican LM, Wurtz RH. Reversible inactivation of monkey superior colliculus. II. Maps of saccadic deficits. J Neurophysiol 79: 2097-2110, 1998.

Rivaud S, Muri RM, Gaymard B, Vermersch AI, Pierrot-Deseilligny C. Eye movement disorders after frontal eye field lesions in humans. Exp Brain Res 102: 110-120, 1994.

Rizzolatti G, Matelli M, Pavesi G. Deficits in attention and movement following the removal of postarcuate (area 6) and prearcuate (area 8) cortex in macaque monkeys. Brain 106: 655-673, 1983.

Robinson DA, Fuchs AF. Eye movements evoked by stimulation of frontal eye fields. J Neurophysiol 32: 637-648, 1969.

Schall JD. The neural selection and control of saccades by the frontal eye field. Philos Trans R Soc Lond B Biol Sci 357: 1073-1082, 2002.

Schieber MH. Inactivation of the ventral premotor cortex biases the laterality of motoric choices. Exp Brain Res 130: 497-507, 2000.

Schiller PH, Chou IH. The effects of frontal eye field and dorsomedial frontal cortex lesions on visually guided eye movements. Nat Neurosci 1: 248-253, 1998.

Schiller PH, True SD, Conway JL. Deficits in eye movements following frontal eye-field and superior colliculus ablations. J Neurophysiol 44: $1175-1189,1980$.

Schlag J, Dassonville P, Schlag-Rey M. Interaction of the two frontal eye fields before saccade onset. J Neurophysiol 79: 64-72, 1998.

Schmahmann JD, Pandya DN. Anatomic organization of the basilar pontine projections from prefrontal cortices in rhesus monkey. J Neurosci 17: 438-458, 1997.

Schnyder H, Reisine H, Hepp K, Henn V. Frontal eye field projection to the paramedian pontine reticular formation traced with wheat germ agglutinin in the monkey. Brain Res 329: 151-160, 1985.

Schwartz EL. Computational anatomy and functional architecture of striate cortex: a spatial mapping approach to perceptual coding. Vision Res 20: 645-669, 1980.

Segraves MA. Activity of monkey frontal eye field neurons projecting to oculomotor regions of the pons. J Neurophysiol 68: 1967-1985, 1992.

Segraves MA, Goldberg ME. Functional properties of corticotectal neurons in the monkey's frontal eye field. J Neurophysiol 58: 1387-1419, 1987.

Segraves MA, Park K. The relationship of monkey frontal eye field activity to saccade dynamics. J Neurophysiol 69: 1880-1889, 1993.

Seidemann E, Arieli A, Grinvald A, Slovin H. Dynamics of depolarization and hyperpolarization in the frontal cortex and saccade goal. Science 295: 862-865, 2002.

Shi D, Friedman HR, Bruce CJ. Deficits in smooth-pursuit eye movements after muscimol inactivation within the primate's frontal eye field. $J$ Neurophysiol 80: 458-464, 1998.

Sommer MA, Tehovnik EJ. Reversible inactivation of macaque frontal eye field. Exp Brain Res 116: 229-249, 1997.

Sommer MA, Wurtz RH. Composition and topographic organization of signals sent from the frontal eye field to the superior colliculus. J Neurophysiol 83: 1979-2001, 2000.

Sommer MA, Wurtz RH. Frontal eye field sends delay activity related to movement, memory, and vision to the superior colliculus. $J$ Neurophysiol 85: 1673-1685, 2001.

Stanton GB, Goldberg ME, Bruce CJ. Frontal eye field efferents in the macaque monkey: I. Subcortical pathways and topography of striatal and thalamic terminal fields. J Comp Neurol 271: 473-492, 1988a.

Stanton GB, Goldberg ME, Bruce CJ. Frontal eye field efferents in the macaque monkey: II. Topography of terminal fields in midbrain and pons. $J$ Comp Neurol 271: 493-506, 1988b.

Tanaka M. Inactivation of the central thalamus delays self-timed saccades. Nat Neurosci 9: 20-22, 2006.

Tanaka M. Cognitive signals in the primate motor thalamus predict saccade timing. J Neurosci 27: 12109-12118, 2007. 
Tanaka M, Fukushima K. Neuronal responses related to smooth pursuit eye movements in the periarcuate cortical area of monkeys. J Neurophysiol 80: 28-47, 1998.

van der Steen J, Russell IS, James GO. Effects of unilateral frontal eye-field lesions on eye-head coordination in monkey. J Neurophysiol 55: 696-714, 1986.

Weinrich M, Wise SP. The premotor cortex of the monkey. J Neurosci 2: 1329-1345, 1982.

Weinrich M, Wise SP, Mauritz KH. A neurophysiological study of the premotor cortex in the rhesus monkey. Brain 107: 385-414, 1984.
White JM, Sparks DL, Stanford TR. Saccades to remembered target locations: an analysis of systematic and variable errors. Vision Res 34: 79-92, 1994.

Wurtz RH, Goldberg ME. Activity of superior colliculus in behaving monkey. IV. Effects of lesions on eye movements. J Neurophysiol 35: 587-596, 1972.

Xiong G, Hiramatsu T, Nagao S. Corticopontocerebellar pathway from the prearcuate region to hemispheric lobule VII of the cerebellum: an anterograde and retrograde tracing study in the monkey. Neurosci Lett 322: 173-176, 2002.

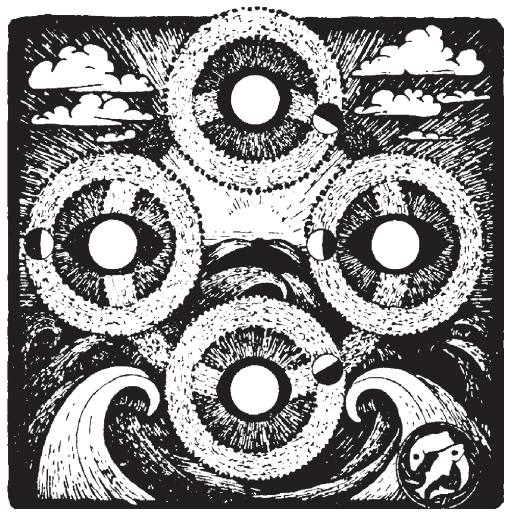

